Portland State University

PDXScholar

$11-22-2019$

\title{
Cortical Thickness Comparison of ASD and TD Subjects
}

Jonathan Rigoberto Uriarte-Lopez

Portland State University

Follow this and additional works at: https://pdxscholar.library.pdx.edu/honorstheses Let us know how access to this document benefits you.

\section{Recommended Citation}

Uriarte-Lopez, Jonathan Rigoberto, "Cortical Thickness Comparison of ASD and TD Subjects" (2019). University Honors Theses. Paper 813.

https://doi.org/10.15760/honors.832

This Thesis is brought to you for free and open access. It has been accepted for inclusion in University Honors Theses by an authorized administrator of PDXScholar. Please contact us if we can make this document more accessible: pdxscholar@pdx.edu. 


\title{
Cortical Thickness Comparison of ASD and TD Subjects
}

By

Jonathan Rigoberto Uriarte-Lopez

An undergraduate honors thesis submitted in partial fulfillment of the

\author{
Requirements for the degree of \\ Bachelor of Science (B.S.)
}

In

University Honors

And

Biochemistry

Thesis Adviser

Damien A. Fair, PA-C, Ph.D.

Oregon Health and Science University

Portland State University

In collaboration with

Oregon Health and Science University

2019 


\section{Table of Contents}

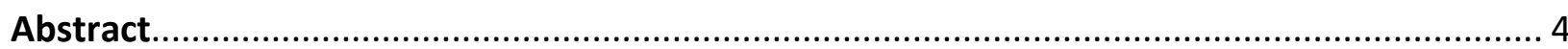

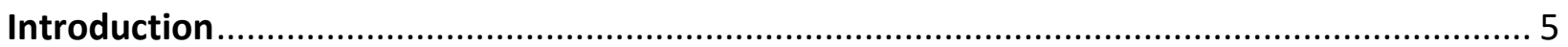

Main characteristics of $A S D$............................................................................................ 5

Symptoms associated with each characteristic ............................................................... 5

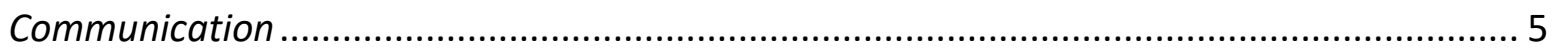

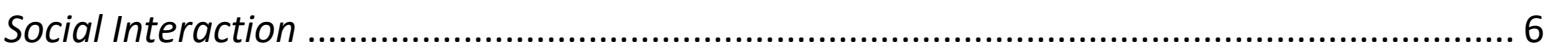

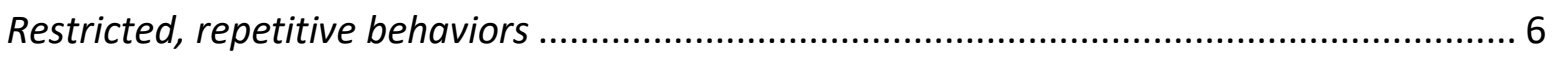

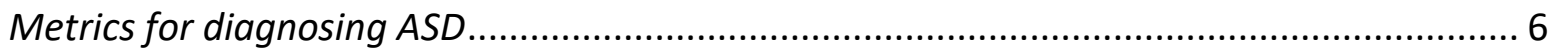

Difficulty defining a disorder due to symptom overlap .................................................. 8

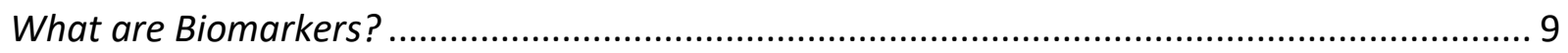

MRI can be used to noninvasively study the brain ............................................................. 9

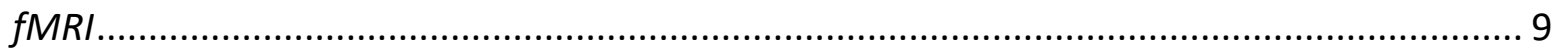

The complexity of MRI studies ................................................................................. 10

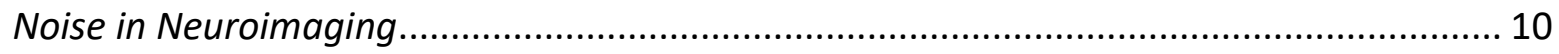

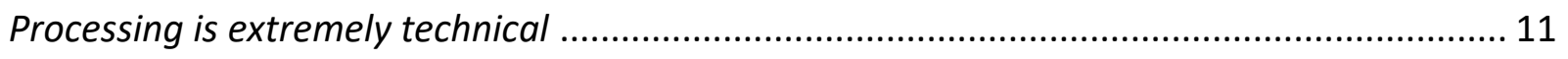

No clear agreement on brain biomarkers associated with ASD ........................................ 12

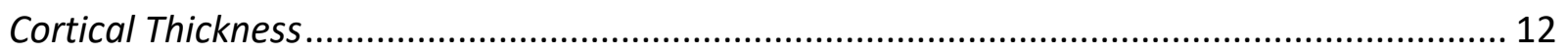


International consortium

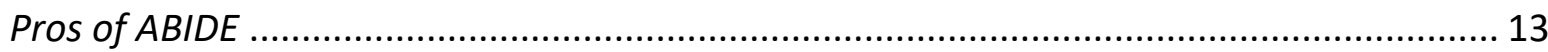

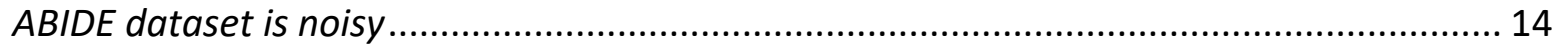

Inconsistencies of the ABIDE dataset for cortical thickness analysis ................................ 14

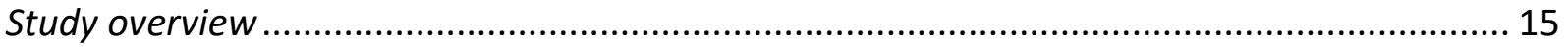

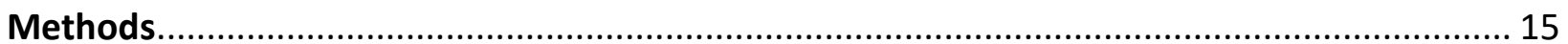

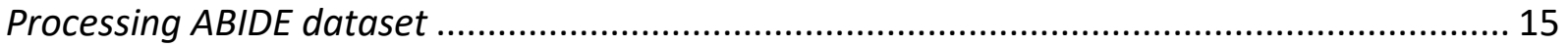

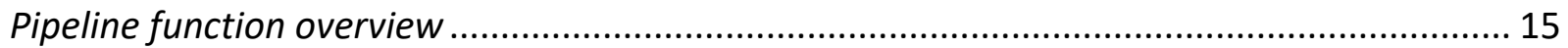

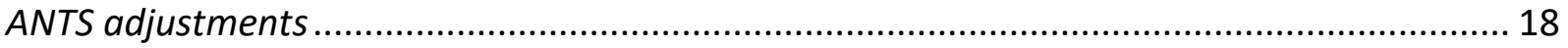

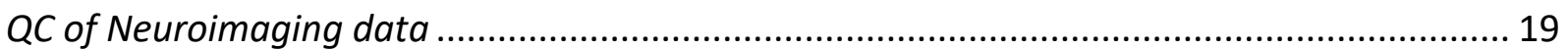

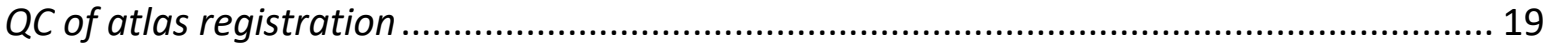

QC of segmentation in structural data ....................................................................... 21

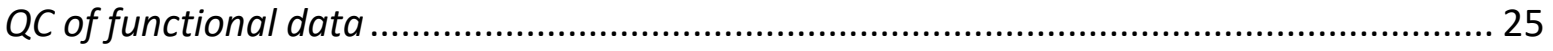

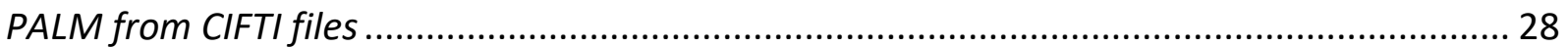

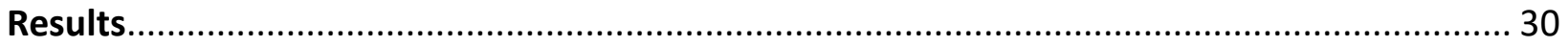

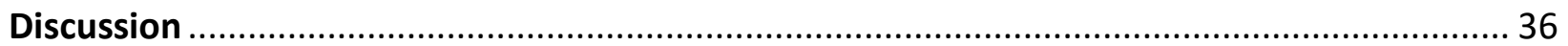

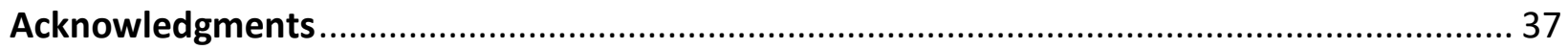

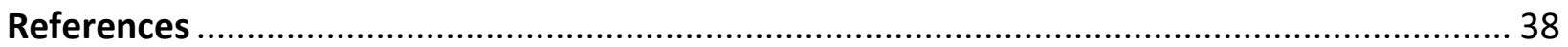




\section{Abstract}

With 1 out of every 59 adolescents diagnosed with autism spectrum disorder (ASD), more research interest has been dedicated to studying why ASD individuals experience their symptoms of restricted and/or repetitive behaviors, and deficits in social interaction and communication. ASD can only be diagnosed by psychiatric evaluation of behavior, and there is a lack of reliable biomarkers for ASD to verify the diagnosis. To potentially find a reliable biomarker, we compared cortical thickness in 911 ASD subjects and 999 controls from the Autism Brain Imaging Data Exchange (ABIDE) dataset. The dataset was processed using the Developmental Cognition and Neuroimaging (DCAN) Labs modified version of the Human Connectome Project (HCP) pipeline. Permutation Analysis of Linear Models (PALM) was used to compare the difference of cortical thickness between ASD and control subjects. An increase of cortical thickness was found in the visual and somatomotor cortex of ASD subjects. Specific differences were found in the left superior parietal $\left(p=0.0565\right.$; cluster size $\left.=202.75 \mathrm{~mm}^{2}\right)$, left occipital lobe $\left(p=0.0861\right.$; cluster size $\left.=175.13 \mathrm{~mm}^{2}\right)$, left temporal lobe $(p=0.0582$; cluster size $\left.=202.76 \mathrm{~mm}^{2}\right)$, and the right temporal lobe $\left(p=0.0274\right.$; cluster size $\left.=265.5 \mathrm{~mm}^{2}\right)$. Future directions to discover reliable biomarkers for ASD will involve exploring the correlation between cortical thickness and behavior, improving neuroimaging protocols for acceptable data acquisition, and using different datasets to further validate biomarkers. 


\section{Introduction}

Main characteristics of ASD

Autism Spectrum Disorder (ASD) is a neurodevelopmental disorder. The estimated prevalence of ASD in the United States is about 1 out of every 59 children (Baio et al., 2018). Characteristics of people with ASD include social deficits and communication difficulties, along with restricted and/or repetitive behaviors (Ousley \& Cermak, 2014). The new edition of the DSM, called the DSM-5, has slightly modified criteria to improve clinical practice. Under the new revision, for someone to be diagnosed with ASD they must show evidence of difficulties in social communication, restricted and/or repetitive behaviors, or unusual sensory-motor behaviors. If an individual has one or more other psychological disorders, the symptoms of those disorders can overlap with ASD symptoms and it can be difficult to distinguish them at times. Diagnosing with ASD based on reliance on symptoms rather than biological diagnostics can lead to misdiagnoses (Frazier et al., 2012).

Symptoms associated with each characteristic

Communication, social interaction, and restricted, repetitive behaviors are the three main contexts for ASD diagnosis. The severity of these symptoms varies from individual to individual. The symptoms can be further defined using the DSM-5 (American Psychiatric Association, 2013).

\section{Communication}

Adolescents with ASD have a high likelihood of experiencing receptive, expressive, vocabulary, and grammatical language impairments. These impairments include limitations in 
language pragmatics or the ability to use language in social adaptation. These symptoms and experiences make it difficult for those with ASD to communicate in a variety of social contexts such as recognizing social cues or interpreting social messages (Tager-Flusberg \& Caronna, 2007).

\section{Social Interaction}

Social interaction skills are important for developing, maintaining, and understanding relationships. ASD individuals may experience symptoms of poor eye contact, and failure to initiate social interactions. In social settings, they may experience difficulty in using and understanding verbal and nonverbal communication to navigate the social environment (Matson, Matson, \& Rivet, 2007).

Restricted, repetitive behaviors

Patterns of narrow and abnormal interests are often presented in ASD individuals. It is common to see highly restricted and fixated interests that are defined as abnormal in intensity or focus. An example of this would be a strong attachment to objects or specific activities of interest. Patterns of repetitive motor movements can be recognized through the abnormal use of objects or speech. This can be shown through an ASD diagnosed individual being more interested in spinning the wheels of a car than driving it around like normal play (Johnson et al., 2007).

Metrics for diagnosing ASD

Early diagnosis and detection of ASD are important to provide early intervention and improve long-term outcomes; however, neurodevelopmental disorders are difficult to diagnose at an early age. This inherent difficulty of early diagnosis is compounded by the fact that there 
are no reliable biomarkers for ASD. The clinician must be able to diagnose ASD in suspected individuals based on their behavior and characteristics. To support the clinician, the diagnosis of ASD involves using the Autism Diagnostic Interview-Revised (ADI-R) and the Autism Diagnostic Observation Schedule-General (ADOS-G), two diagnostic metric tools used to analyze and assess the diagnosis of ASD based on behavior.

The ADI-R is a standardized, semi-structured, clinical review for caregivers of children and adults who may have a possible ASD diagnosis (Lord, Rutter, \& Couteur, 1994). This diagnostic tool is used to assess the abilities of social interactions, communication and language capabilities, and for the analysis of restricted and repetitive actions or interests. The ADI-R is an effective tool to give the clinician the developmental history of the individual, but it is not the best tool to diagnose a suspected individual at a current time point.

The ADOS-G is also a semi-structured, standardized assessment much like the ADI-R, but it is much more rigorous and time-consuming. The suspected individual undergoes an assessment through different testing sections of social interaction, communication, play, and imaginative use of materials. The reason why the ADOS-G is the most accurate metric is due to its reliability, consistency, ability to classify ASD or non-ASD disorders, analysis of ASD severity, and its test-retest ability (Lord, C., Rutter, M., DiLavore, P., Risi, S., \& Gotham, 2012). This three-hour-long metric is great for measuring the individual at a single time point, but it does not give the clinician developmental history.

Field trials of the DSM-5 were conducted to validate its diagnostic capabilities for ASD. The trials underwent two phases with a test-retest design to ensure accuracy. Phase I of the trials showed that clinical ASD diagnosis is not as reliable as semi-structured diagnostic tools 
such as the ADI-R or the ADOS-G. As many as $12 \%$ of ASD individuals, a large portion of them being females, were undiagnosed. This is due to the algorithm used to assess an ASD diagnosis. Compared to the DSM-4, the DSM-5 had higher specificity (DSM-5 $=0.97, \mathrm{DSM}-4=0.86$ ) but lower sensitivity (DSM-5 $=0.81, \mathrm{DSM}-4=0.95)$. Adjusting the algorithm by removing one symptom criterion increased sensitivity (Original $=0.81$, Adjusted $=0.93$ ) while maintaining a similar specificity (Original $=0.97$, Adjusted $=0.95$ ). Improvements to the sensitivity will allow a more consistent ASD diagnosis for clinicians. The DSM field trials showed that the DSM-5 has limitations in the proper identification of ASD (Frazier et al., 2012).

Despite the use of the DSM-5, ADI-R, and the ADOS-G as diagnostic metrics, the clinician can still be susceptible to misdiagnosis of ASD from combined characteristics of other mental disorders.

\section{Difficulty defining a disorder due to symptom overlap}

The symptoms and characteristics of ASD overlap with other mental disorders, and comparative studies between multiple mental disorders are rare. Individuals with ASD have an increased risk of experiencing one or more co-occurring psychiatric conditions (Rosen, Mazefsky, Vasa, \& Lerner, 2018). Attention-deficit/hyperactivity disorder (ADHD) is another neurodevelopmental disorder that is characterized by attention difficulties, hyperactivity, and impulsivity. Children with ASD have a high co-occurrence of ADHD, and a study group of individuals diagnosed with ASD and ADHD reveals similarities between characteristics of both disorders (Craig et al., 2015). Anxiety and depression are also represented at elevated rates among individuals diagnosed with ASD (Strang et al., 2012). The prevalence and overlap of ASD 
with other mental disorders make it difficult to accurately diagnose ASD based on a clinical review of an individual's characteristics alone.

\section{What are Biomarkers?}

Biomarkers are used to measure and evaluate substances, structures, or processes within the body to determine the incidence and outcome of disease (Strimbu \& Tavel, 2010). Once a biomarker is properly assessed it can be used to verify a diagnosis of a disease or disorder. Neuroimaging biomarkers and genetic or molecular profiling are the two types of biomarkers that can be used to validate an ASD diagnosis.

$M R I$ can be used to noninvasively study the brain

Magnetic resonance imaging (MRI) is an instrument that allows researchers to noninvasively study the structural and functional characteristics of the brain. This is done with the use of advanced neuroimaging analysis techniques that can perform segmentation to detect and analyze specific tissue types, and further parcellation can be performed to study specific regions of interest (ROI) of the brain (Giedd et al., 2009). Segmentation is used to study the separation of gray matter (GM), white matter (WM), and cerebral spinal fluid (CSF). $f M R I$

Functional MRI (fMRI) is a technique used to measure brain activity. The technique utilizes the fact that neural activity increases blood flow without an associated increase in oxygen metabolism. Any difference between the ratio of blood flow and oxygen metabolism can be detected via MRI. This signal is called blood oxygen level dependence (BOLD) (Raichle \& Mintun, 2006). An increase in the BOLD signal is correlated with cortical activation because more oxygenated blood is supplied to the brain for immediate metabolic activity. 
The complexity of MRI studies

MRI machines are expensive and require extensive technical skills to operate. When considering the challenges in subject recruitment and retention, it is difficult and expensive to have many subjects for neuroimaging studies. Low subject turnout results in a low sample size which reduces the statistical power, reliability, and the replicability of neuroimaging findings (Turner, Paul, Miller, \& Barbey, 2018). fMRI has become a popular tool to study the human brain. However, the statistical methods used in $\mathrm{fMRI}$ studies are rarely validated using real data. This, in turn, can cause the possibility of reporting false positives or false negatives in neuroimaging studies (Eklund, Nichols, \& Knutsson, 2016).

Noise in Neuroimaging

The MRI signal is susceptible to various sources of noise such as motion within the scanner, respiration of the participant, and background noise. All these factors can disrupt the signal to noise ratio (SNR). Motion contaminated, or "noisy", MRI scans have a low SNR which leads to poor data quality and limited usage for research. Patient head movement within the scanner can cause shifts between signals. If the shifts are excessive then they can disrupt the MRI machine's ability to collect proper frame acquisition, and image intensity thus lowering the SNR. "Noisy" scans can lead to inaccurate brain size estimates, poor anatomical characterization, and skewed measurements of white and gray matter delineation (Savalia et al., 2017). For fMRI scans, oxygenated blood measurements are corrupted and can generate skewed results for neurophysiological related scans. The only correction is to realign the headin-space position along the xyz-axes and their respective rotational displacements (Siegel et al., 
2014). This requires precise realignment which can be very technical and difficult to perform but must be done to salvage "noisy" scans.

If the motion is too excessive then this leads to the formation of motion artifacts which renders the subject's scan unusable. MRI scanners obtain images of the brain by acquiring multiple frames or slices. If there is too much distance between the acquisition of one frame to the next, this results in distance-dependent motion artifacts (Ciric et al., 2017). This drastically affects brain structure and functional connectivity outputs. Examples of motion artifacts are shown in the methods section.

\section{Processing is extremely technical}

In our study, the MRI scans we obtained are processed with the latest state of the art human connectomes project (HCP) minimal preprocessing pipeline (Glasser et al., 2013). Highresolution T1w and T2w scans are required by the pipeline to perform surface reconstruction and generate surface images. This adds another barrier in proper data acquisition since highresolution scans require a stronger MRI machine, such as a 3T scanner, for improved data quality before they can be processed with the HCP pipeline.

The Developmental Cognition and Neuroimaging (DCAN) labs developed a modified HCP pipeline to solve the issue of noisy MRI data. In order to make the HCP pipeline more usable for a wide variety of MRI scans from different scanners, the HCP pipeline was modified using the advanced normalization tools (ANTS) algorithm to improve scan quality (Avants, Tustison, \& Song, 2009). More details will be presented in the methods section. These modifications provide an avenue to increase the signal to noise ratio (SNR) in MRI and to allow the technique to detect biomarkers in neurodevelopmental disorders, such as ASD. 
No clear agreement on brain biomarkers associated with ASD

The longitudinal neurodevelopment of ASD is not very well understood. ASD may manifest itself differently in people of different ages, which can result in conflicting accuracy for diagnostic results. Our area of focus will be to assess cortical thickness differences between ASD and typically developing (TD) subjects in adolescents and adults.

\section{Cortical Thickness}

Cortical thickness is a brain morphometric that measures the thickness or volume of the cerebral cortex. The cerebral cortex is an important part of the brain that plays a critical role in consciousness. Studies have shown that there is a difference in cortical shape between children with simplex ASD and TD children. Bilateral differences were discovered in sulcal depth in the restricted portions of the anterior-insula and frontal-operculum and the temporoparietal junction has been identified (Dierker et al., 2015).

There are many conflicting studies when it comes to the analysis of cortical thickness differences between the two subgroups (Amaral, Schumann, \& Nordahl, 2008). There is evidence to support the case of increased cortical thickness throughout the entire brain (Hardan, Muddasani, Vemulapalli, Keshavan, \& Minshew, 2005), and other studies instead show cortical thinning in multiple brain regions (Hadjikhani, Joseph, Snyder, \& Tager-Flusberg, 2006). Age is another variable that can skew the results of ASD neuroimaging studies. Studies using younger subjects reported increases in brain volume in ASD individuals, but others that use an older cohort report no changes in brain volume (Pagnozzi, Conti, Calderoni, Fripp, \& Rose, 2018). 
Abnormal early brain volume overgrowth has been detected when comparing the average of 131 ASD to 50 TD subjects that are within the ages of 2-5 years old (Nordahl et al., 2013). This promising finding can support the case of using abnormal cortical thickness measurements as a potential biomarker for ASD. The reason why cortical thickness is not normally used as a biomarker is because of evidence that goes against its usability. The conflicting results have been reported by studies using 89 men with ASD and 89 TD men between the ages of 18-43 (Ecker, 2012), and in another study where they had 51 ASD men and 49 TD men between the ages 30-75 (Koolschijn \& Geurts, 2016). To answer this, we require a large dataset to avoid results being affected by underpowered statistical analysis from low sample sizes common in neuroimaging studies.

\section{International consortium}

To address the issue of low sample sizes in neuroimaging studies, Dr. Di Martino and her colleagues came together to form the Autism Brain Imaging Data Exchange (ABIDE) (Di Martino et al., 2014). ABIDE contains MRI and fMRI neuroimaging scans of 2226 TD and ASD diagnosed subjects. These scans were obtained and shared by 25 international labs, and they have been uploaded to a publicly available open-access online repository. The age range for all participants is 5-64 years old.

Pros of $A B I D E$

ABIDE provides metadata for a large number of subjects with reasonable accessibility. This means that researchers interested in using the ABIDE dataset acquire it directly from the source for their analysis. This international consortium can grow by accepting new labs and thus increasing the total number of ASD and TD subjects. 


\section{ABIDE dataset is noisy}

As mentioned previously, the HCP pipeline has a certain set of criteria for neuroimaging scans to be processed through the pipeline and generate usable neuroimaging scans. If an input scan turns out to be "noisy," then the scan cannot be processed or it will generate an inaccurate brain map depending on the SNR. The cause for some "noisy" scans stems from the fact that many different scans come from many different sites. Each site may use MRI scanners from different manufacturers along with different scanner protocols. This will affect how the metadata is collected at each site, resulting in batch effects or differences between data caused by a disruption in control variables. When the subject's metadata is processed through the computing pipeline there is a possibility that false positives and false negatives may arise affecting analysis of brain ROI because of poor scan quality. A reliable dataset needs to be used to accurately map out the brain and to note differences between ASD and TD subjects. Without a consistent database, the results will be skewed.

Inconsistencies of the ABIDE dataset for cortical thickness analysis

The ABIDE dataset still suffers from inconsistent brain differences of ASD individuals. In a study using this dataset, individuals with ASD showed no apparent brain abnormalities despite having ASD behavioral characteristics. Anatomical insight of the amygdala, hippocampus, cerebellum, and corpus callosum as ROIs show no apparent differences. The accuracy of a multivariate classification using volumetric, thickness, and surface area yielded an accuracy of $<60 \%$ (Haar, Berman, Behrmann, \& Dinstein, 2016). 
Study overview

The large MRI dataset provided by the ABIDE initiative was processed with the DCAN modified HCP pipeline to generate more accurate and precise outputs with ANTS. This dataset was split into two groups: ASD and TD (controls). All other subcategories were ignored. To detect the brain differences between ASD and TD individuals, the Permutation Analysis of Linear Models (PALM) will be used to analyze the reprocessed ABIDE dataset. PALM is experimental software that allows inferences using permutation methods (Winkler, Ridgway, Webster, Smith, \& Nichols, 2014). PALM can carry out multiple testing and comparisons, which makes it possible to do multi-group analysis for a large dataset like ABIDE for multi-group analysis. This is similar to the multi-group analysis used to compare fMRI task data (Eklund, Nichols, \& Knutsson, 2016).

\section{Methods}

\section{Processing ABIDE dataset}

Figure 1 displays how the ABIDE dataset was processed. The ABIDE dataset comes from this online repository (http://fcon 1000.projects.nitrc.org/indi/abide/). Out of the total 2226 subjects available, 1910 were reprocessed successfully. Only the subjects that were successfully reprocessed were used in this analysis.

\section{Pipeline function overview}

Subject data is first processed through the structural pipelines in the order of PreFreeSurfer, FreeSurfer, and finally PostFreeSurfer. fMRI data is processed through the HCP 
Functional Pipelines through the fMRI Volume and surface pipelines. fMRI data wasn't analyzed for this study, but it is available in the processed outputs.

PreFreeSurfer generates structural brain volume from the T1 image and matches that data to a standard brain template, specifically the Montreal Neurological Institute (MNI) template (Evans, Janke, Collins, \& Baillet, 2012) to ensure that brain structure is as consistent as possible. Brainmask calculations are formulated and used to fit the T1 scan with the MNI atlas to generate an atlas registration. Denoising and bias field corrections through ANTS are implemented during PreFreeSurfer to improve data quality. More details will be provided later.

Brain segmentation of gray and white matter along with predefined structures (Surfer.nmr.mgh.harvard.edu/fswiki/) is performed during the FreeSurfer step after atlas registration. This is done by using the cortical surfaces of the brain. They are reconstructed to match the predefined structures noted, and the cortical surfaces are registered to the surface template.

The resulting data from brain segmentation is stored as Connectivity Informatics Technology Initiative File Format (CIFTI) files. This is generated in the PostFreeSurfer step. CIFTI files are the standard file format for neuroimaging scans as they contain surface and volumetric data (https://www.nitrc.org/projects/cifti/). CIFTI files contain "grayordinate" space information used to define the spatial dimensions of the brain. This is done by using cortical surface vertices or subcortical voxels to model grayordinate space (Glasser et al., 2013). 
DCAN-modified HCP Minimal Preprocessing Pipelines

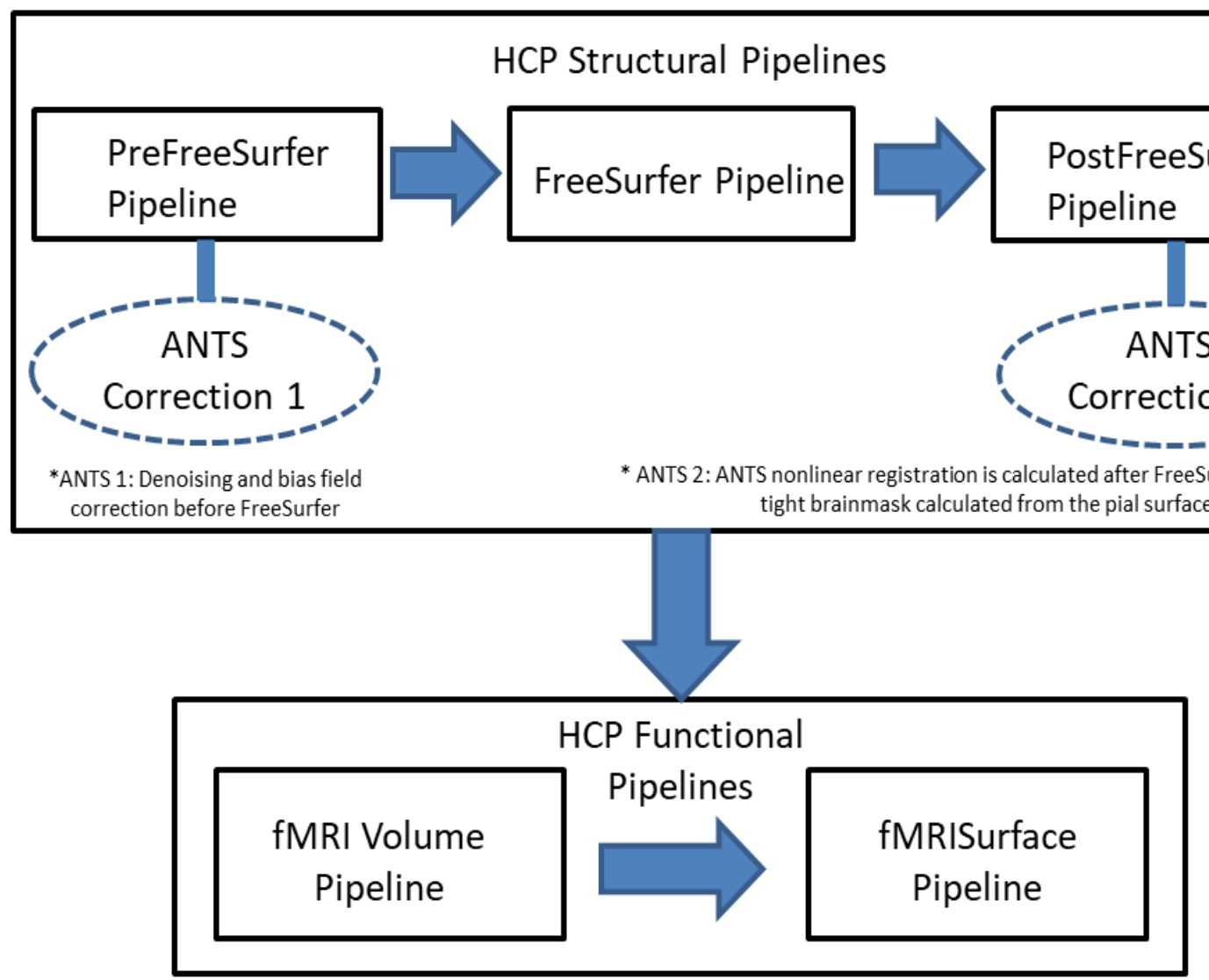

Figure 1: Diagram overview of the modified HCP pipeline derived from (Glasser et al., 2013). HCP structural pipelines are run first, then it is proceeded by the HCP Functional Pipeline, also called the Diffusion Preprocessing Pipeline. During ANTS correction 1, denoising and bias field correction are performed in PreFreeSurfer, prior to typical HCP PreFreeSurfer. In ANTS Correction 2, ANTS nonlinear registration is calculated after FreeSurfer using the tight brainmask calculated from the pial surface in FreeSurfer.

Once CIFTI files are generated, the functional pipelines register fMRI data during the fMRIVolume and fMRISurface stages. The fMRIVolume stage removes spatial distortions, realigns volumes to adjust for subject movement, and reduces bias field, before registering fMRI data to the structural pipeline outputs. Then the fMRISurface pipeline maps the normalized fMRI data to the spatial dimensions of grayordinate space in CIFTI files for proper alignment. 


\section{ANTS adjustments}

The purpose of implementing the ANTS algorithm is to improve MRI scan quality processed through the HCP pipeline by reducing the amount of noise to improve the SNR. Noise can be influenced by background interference or by subject motion within the scanner. An excessive amount of noise can lead to motion artifacts that render the scan useless. ANTS correction 1 is implemented prior to the HCP's PreFreeSurfer, and FreeSurfer pipeline. The algorithm increases the SNR ratio through a process called denoising which filters out the amount of background noise. MRI and $\mathrm{fMRI}$ scans are also susceptible to bias field signals, and ANTS correction 1 denoising is able to remove that as well. This smooth, low-frequency signal is also called intensity inhomogeneity. Bias field errors occur due to the spatial inhomogeneity between the subject and the magnetic field (Despotović, Goossens, \& Philips, 2015). Motion, vibrations, and external noise affect the segmentation of gray and white matter due to the intensities of their respective voxels being distorted, leading to improper delineation. The denoising process decreases the impact of noise affecting the SNR, and potentially decrease or remove artifact errors caused by a bias field signal.

A more accurate pairing between T1 images to the MNI atlas is performed in ANTS correction 2. The nonlinear registration of ANTS correction 2 is performed after FreeSurfer and before the HCP's PostFreeSurfer processing. This is done by using a tight brain mask to increase the precision and accuracy of aligning a T1 scan to the MNI atlas. The tight brainmask is calculated from the pial surfaces recorded in the FreeSurfer step. This better pairing between the T1 scans with the MNI atlas will standardize all subject metadata to ensure that all neurological landmarks and surfaces match up accordingly. ANTS then generates study-specific 
templates. These templates are then skull stripped to remove all bone and leave only brain matter. Delineation of gray and white matter is identified once the segmentations are mapped to the ANTS generated templates. Once the scans are successfully processed, they then undergo a quality control assessment protocol to determine the usability of the dataset.

\section{QC of Neuroimaging data}

Quality control $(Q C)$ is a review process to determine the usability of the dataset. The guidelines are derived from the "Standard Operating Procedure for Quality Assessment," a process developed by the Developmental Cognition and Neuroimaging (DCAN) labs at Oregon Health and Science University (OHSU). The grading criteria uses a three-point system of 1, 2, and 3 to determine the usability of scans. A score of 1 represents usable data, meaning that the scan can be confidently used for analysis. A 2 is given to scans that are probable in terms of data quality because a moderate amount of errors was presented during the QC assessment. If a scan contains too many errors, it is given a 3 . Scans graded with a 3 are not suitable for analysis and should be removed. Once the data has been processed, the quality control guidelines are used to assess the subject's atlas registration, structural data, and functional data.

QC of atlas registration

Atlas registration is an attempt to map the subject's T1 image to an atlas, specifically the MNI atlas as mentioned previously. The quality of the scan is determined by how well the two images overlap. Figure 2 shows an example of a properly matched T1 and atlas. Both images show the matching of the T1 and the atlas. The first image on the left shows the atlas outlines in red on top of the T1 image; the second shows the T1 outlines on top of the atlas. This is done 
to see two different perspectives to accurately grade the images. Proper atlas registration is the first important QC criteria since any errors present in the atlas registration will appear downstream in both the structural and functional scans.

A common error that occurs is protrusions, shown with arrows in figure 3. A protrusion is a misalignment between the $\mathrm{T} 1$ and the $\mathrm{MNI}$ atlas. This can cause improper brain morphology within the structural and functional scans. This can also affect gray and white matter segmentation which may lower the QC grade when assessing the structural scans. Protrusions can go inward or outward, but any signs of protrusions can affect the overall quality of a subject's data.
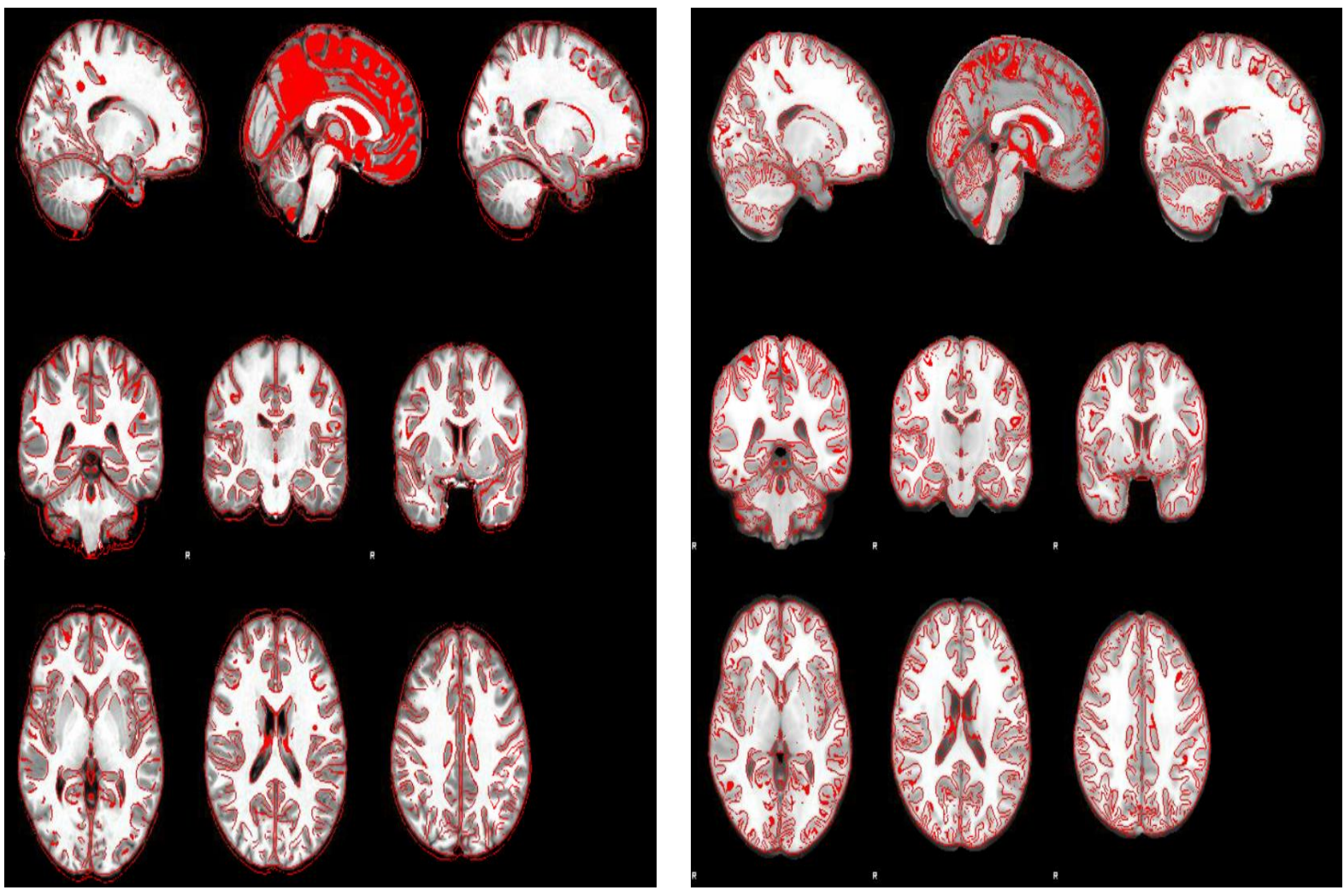

Figure 2: Example of a usable atlas registration (QC score $=1$ ). Atlas in T1 (Left). Panel B: T1 in Atlas (Right) 


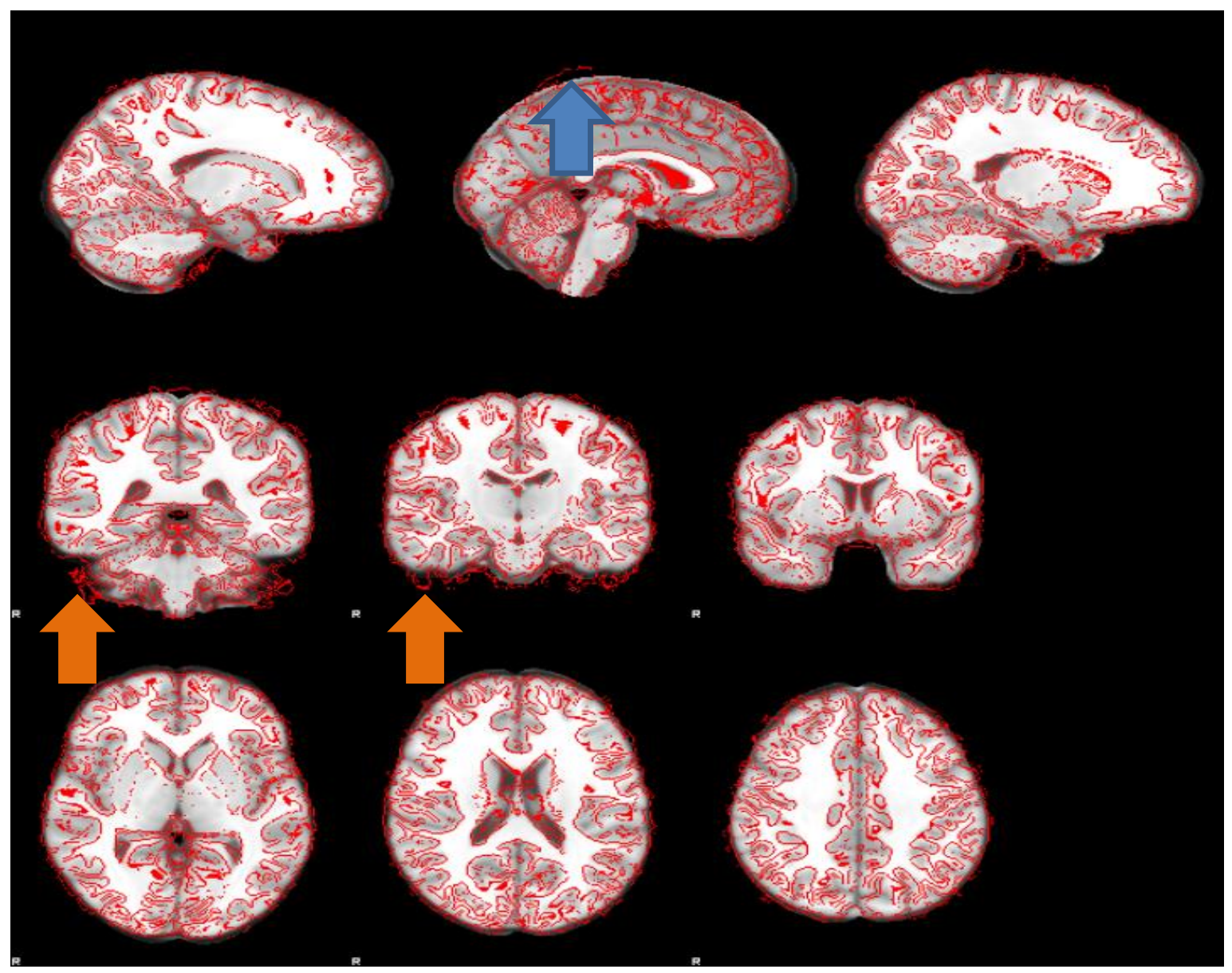

Figure 3: Protrusions present in superior parietal (blue arrow), and in temporal lobes (orange arrows)

QC of segmentation in structural data

QC of structural data is more complex than atlas registration. It requires an assessment of delineation or separation of gray and white matter within the brain, ensuring that the volumetric shape of the brain is consistent and not affected by motion artifacts. White matter is located in the more inner parts of the brain, while gray matter surrounds white matter and is found in the outer layers of the brain.

The structural data can be opened using BrainSprite Viewer as displayed in figure 4 (https://github.com/SIMEXP/brainsprite.js/). This allows the quality controller to examine the 
brain in three dimensions on the $x, y$, and z-axis. White matter is delineated using black lines while the gray matter is delineated using red lines (figure 4).

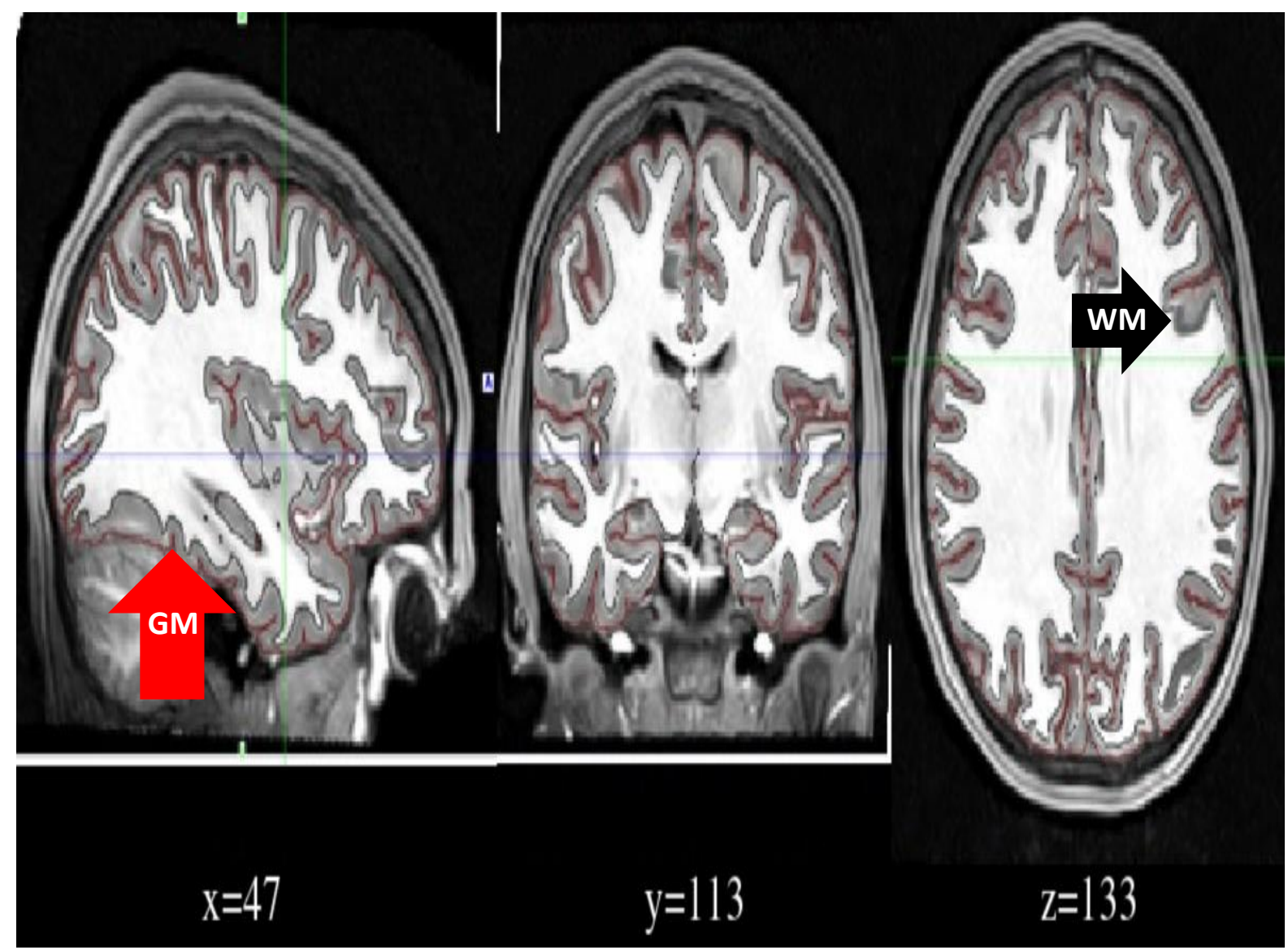

Figure 4: Example of Structural Data in BrainSprite Viewer. Gray matter delineated with red lines (red arrow), and white matter delineated with black lines (black arrow)

The advantage of using BrainSprite Viewer is the ability to look through multiple slices of the scan, to see if it improves when an error appears. For minor errors, the subject is given a 10-frame window to improve the error. For example, if an error occurs at $x=83$, then the subject has until frame $x=93$ or $x=73$ to allow the error to go away. This may save subjects with 
usable data from being deemed unusable. Subjects that experience more severe errors such as motion artifacts cannot be given the 10 -frame grace.

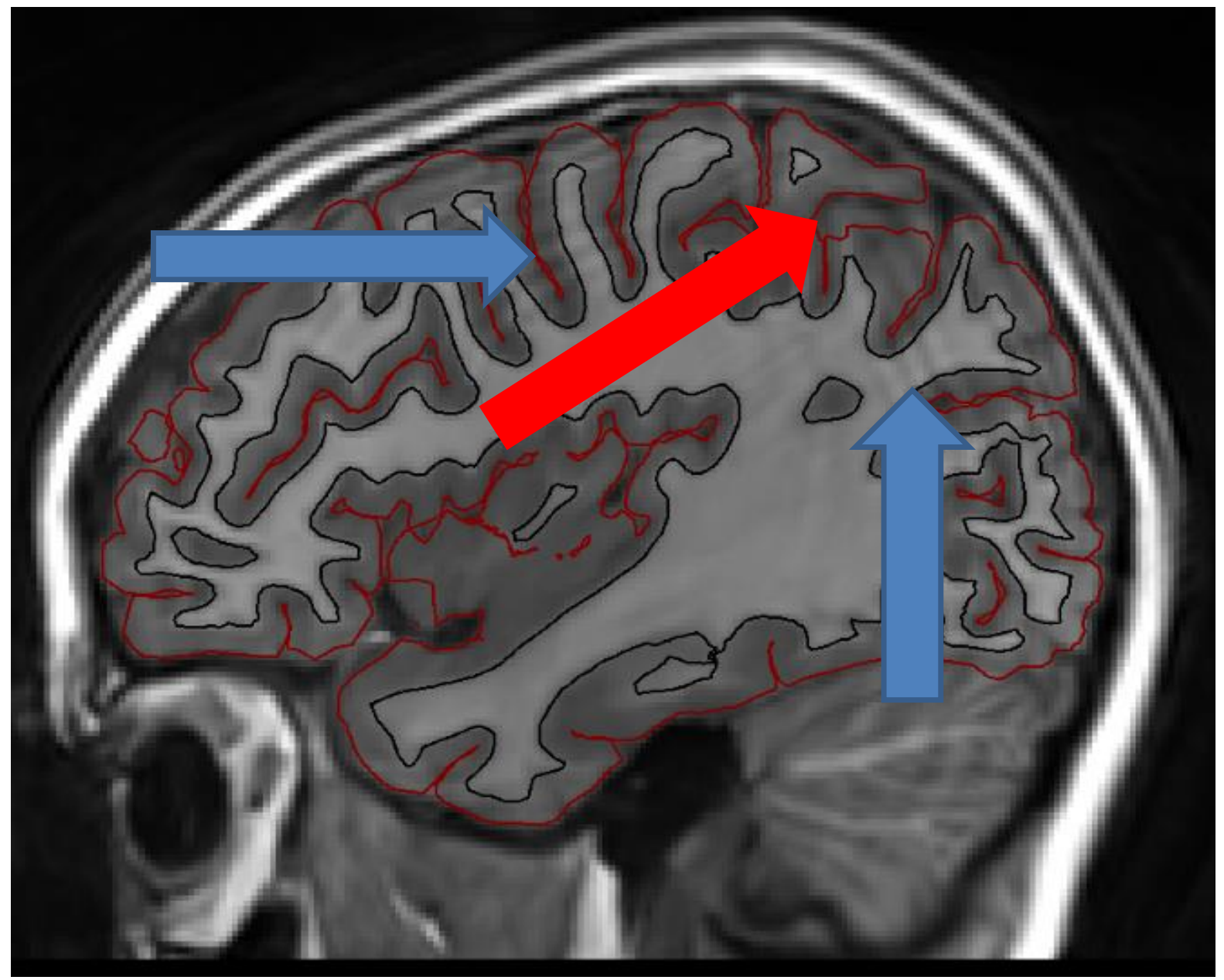

Figure 5: Example of ringing, shown as blue arrows. This can disrupt delineation in areas pointed out by the red arrow

Motion artifacts are more common errors that can affect delineation that is caused due to movement by the subject within the scanner during data acquisition. Ringing is shown as ripple waves throughout the brain, making delineation more difficult (figure 5).

A more serious motion artifact is warping which is seen in figure 6. Warping can "pull" the brain and the skull causing this deformed look. Any presence of warping results in a failed 
QC score, meaning that this subject's data is unsuitable for analysis. All the errors mentioned here would not be given the 10 -frame grace.

Other errors include blurriness and sawtooth lines. Blurriness is a way to describe an image with low pixel clarity. This won't affect most scans, but if excessive enough can affect delineation and make QC difficult. The lines used to delineate gray and white matter should be as straight and/or as curved as possible. At times there can be errors with the line themselves, making these jagged lines called sawtooth lines.

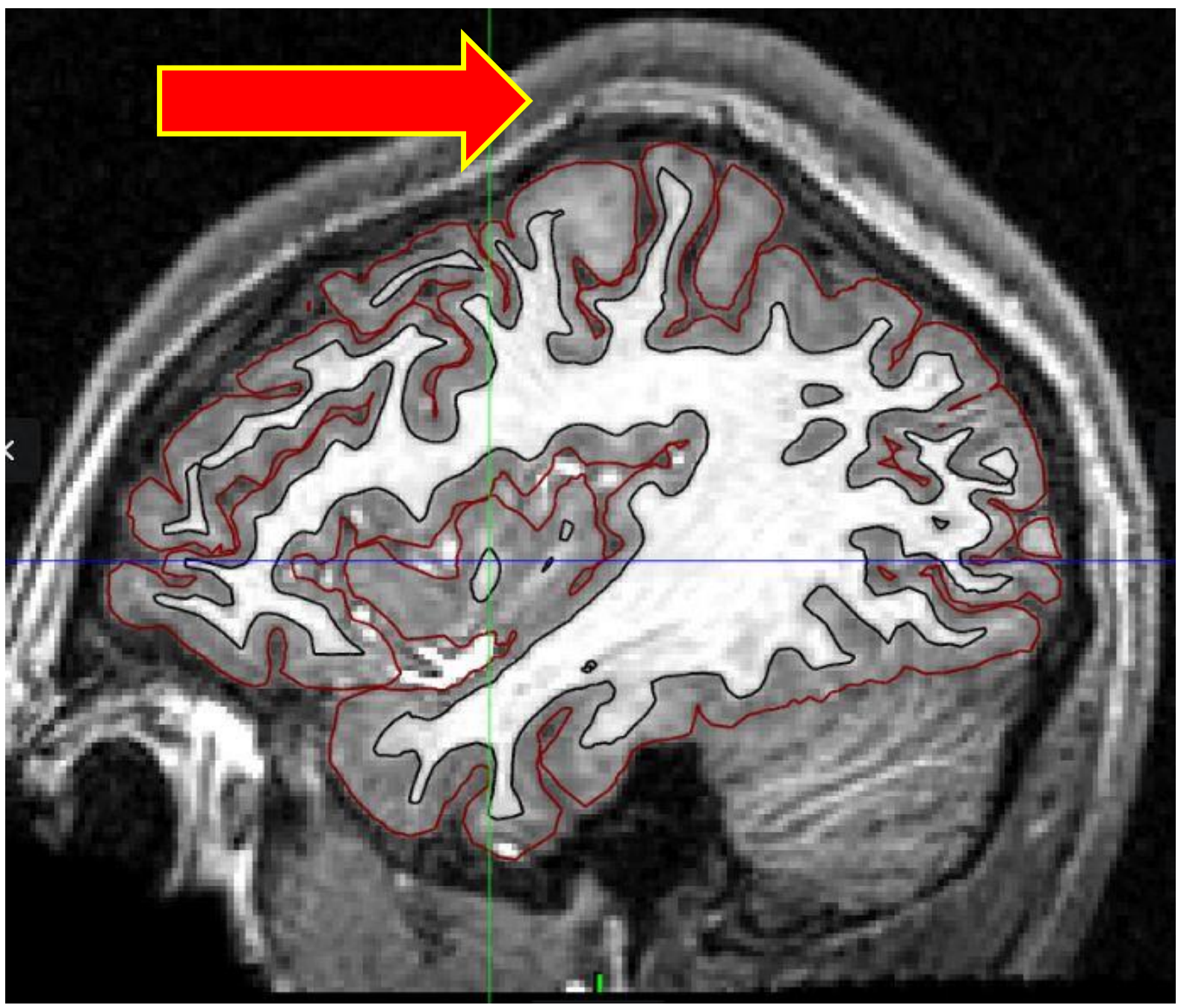

Figure 6: Warping shown in superior parietal portion of subject (red arrow) 


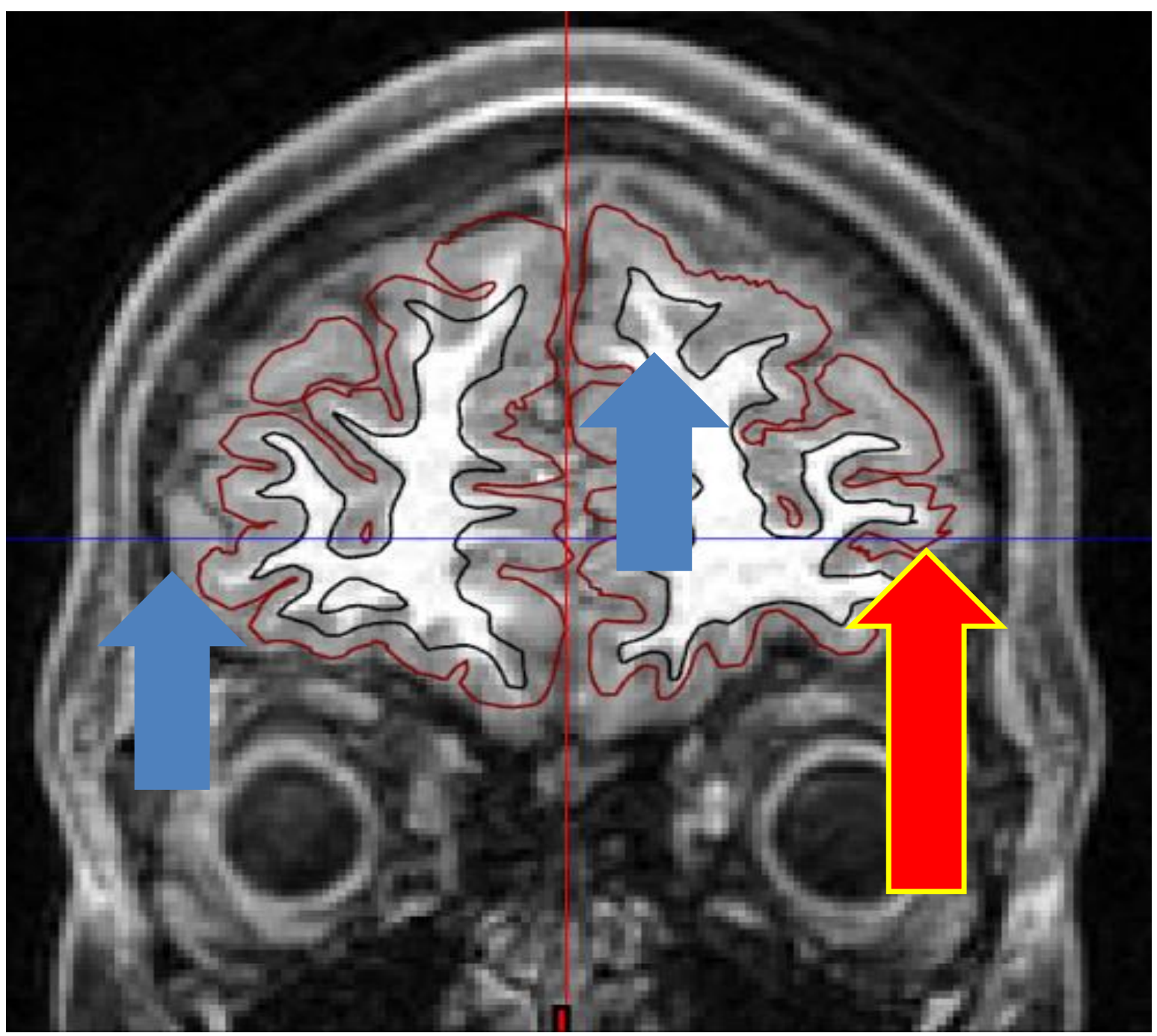

Figure 7: An unusable scan (QC score $=3$ ) of a blurry subject with improper delineation (blue arrows). Sawtooth denoted with red arrow

\section{QC of functional data}

The last set of data to QC is functional data where the quality of fMRI data is assessed.

The main purpose of this QC section is to ensure that the functional scans and the structural scans are properly registered to one another. Figure 8 shows an example of a usable scan when the functional and structural scans register correctly (figure 8). 


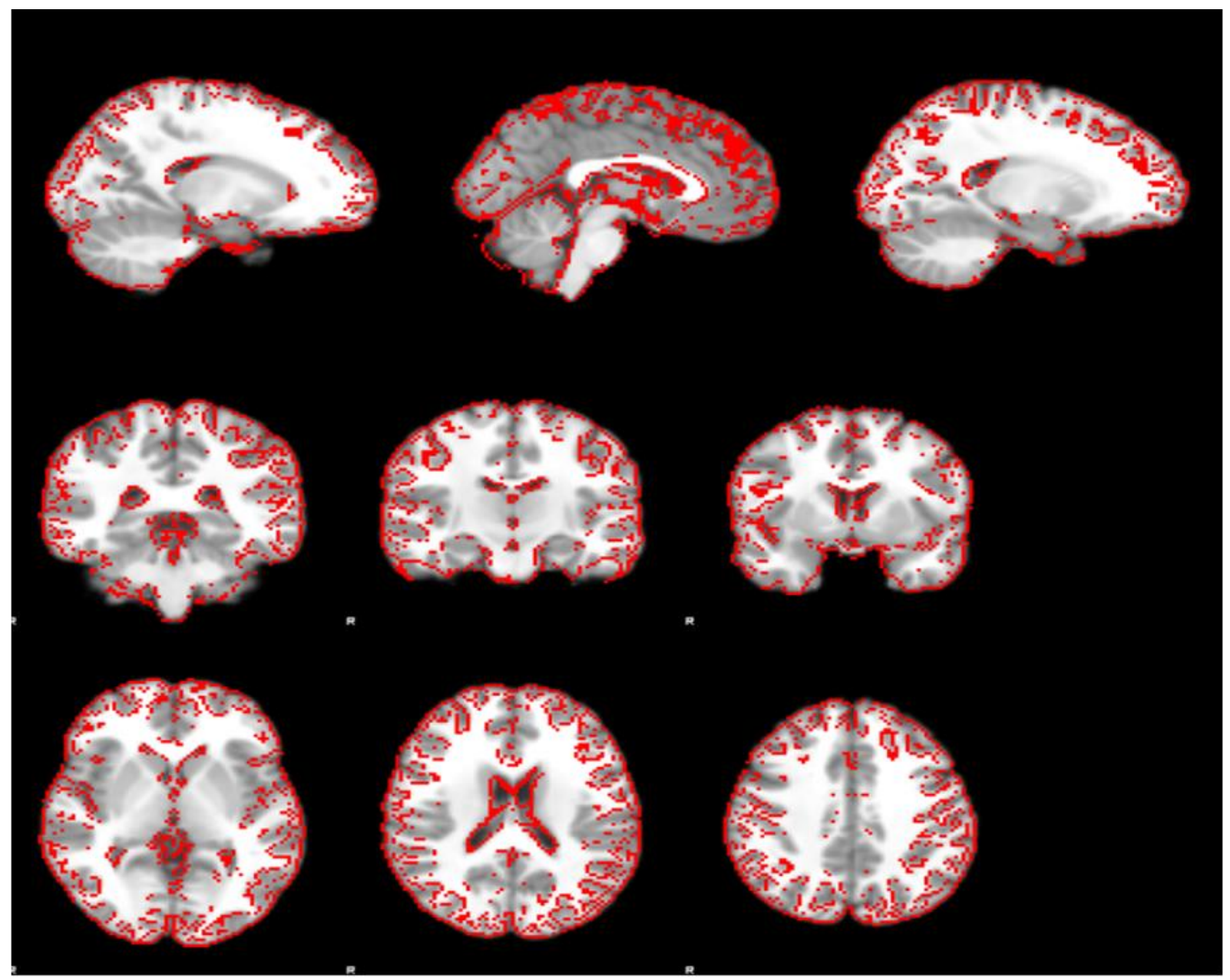

Figure 8: A usable (QC score $=1$ ) fMRI scan with minor signal dropout in temporal region

Many types of errors can be presented. Field of view (FOV) errors describe portions of the brain where there are large areas of BOLD signal dropout or missing outlined regions of the brain (figure 9). Any FOV error that misses more than $10 \%$ of the brain drops the scan's QC score from usable (1) to questionable (2). If more than $50 \%$ of the brain is missing, the scan is automatically given a QC score of unusable (3). Motion artifacts are shown as horizontal lines (figure 10). The presence of motion artifacts can affect the QC grade. A couple of these lines can be present in a scan and can still be graded as usable (1). If a handful of these horizontal 
lines are presented in many sections of the brain, then the scan is graded as questionable (2).

An excessive amount of these horizontal lines presented throughout the scan will result in a QC grade of unusable (3). The more motion artifact presented within the scan, the lower the overall quality. Figure 10 shows an example of a moderate amount of these horizontal lines, and the scan displayed is graded as probable (2). These horizontal lines are secluded in only specific areas of the brain. While this amount of horizontal lines is not ideal, it is not enough to deem the scan as unusable (3).

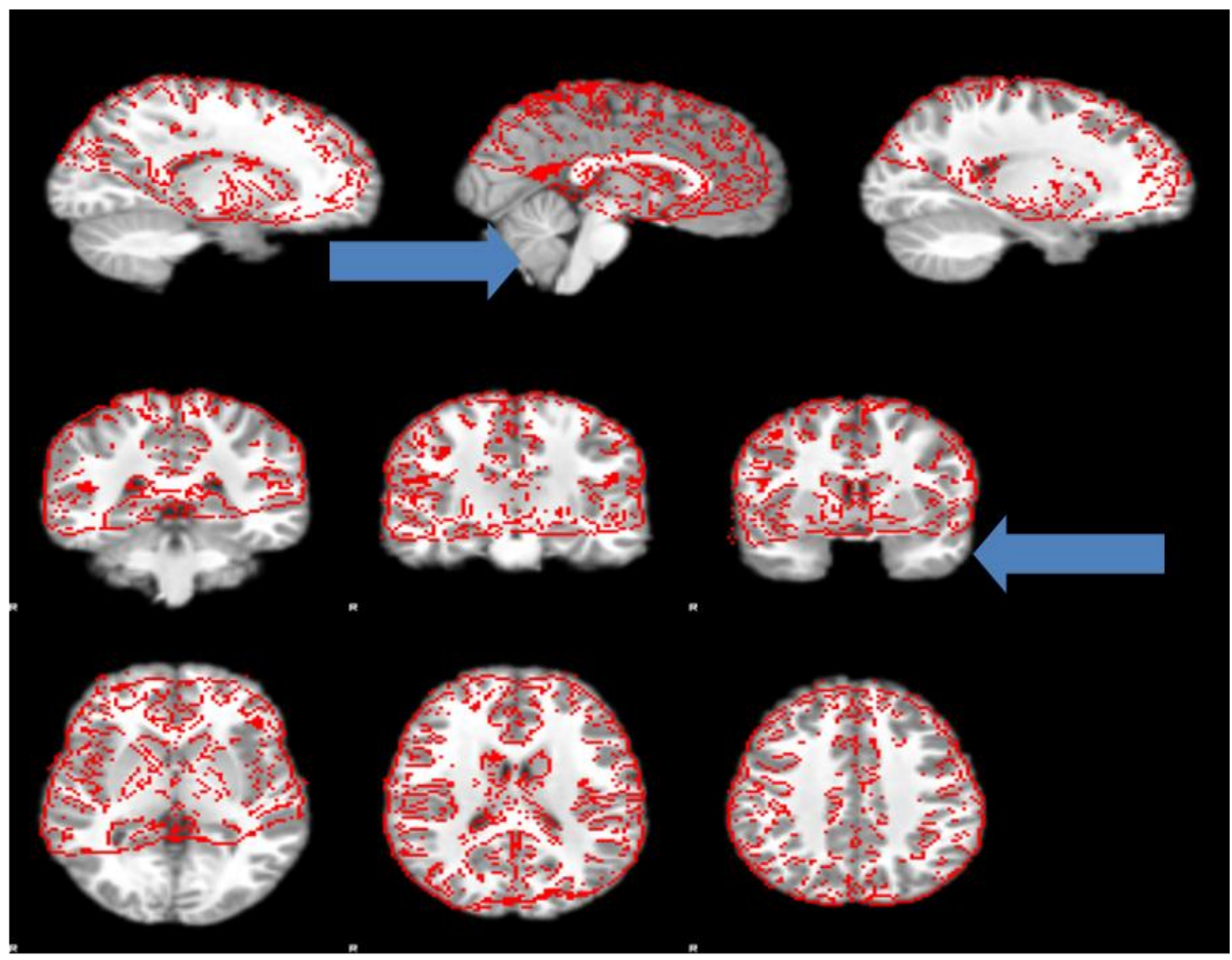

Figure 9: A scan with excessive FOV errors denoted with blue arrows (QC score $=3$ ) 


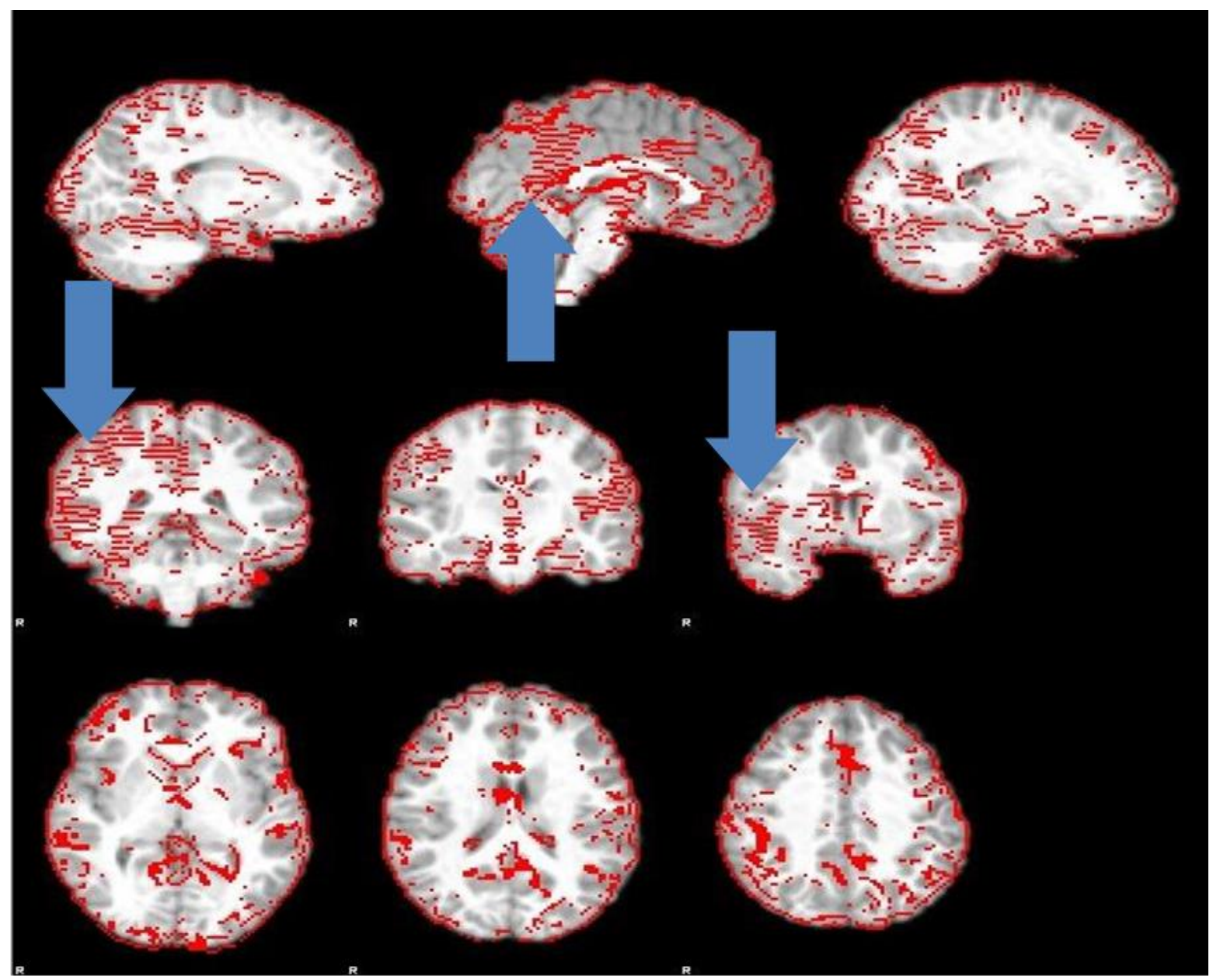

Figure 10: A rest scan with motion artifact denoted with blue arrows $(Q C$ score $=3$ )

\section{PALM from CIFTI files}

The formulated CIFTI files will be used to generate cortical thickness outputs with the use of the Permutation Analysis of Linear Models (PALM) software (figure 11; https://fsl.fmrib.ox.ac.uk/fsl/fslwiki/PALM). This tool can work with volumetric and surfacebased formats provided by the CIFTI files. PALM can undergo multiple testing and comparisons which work effectively for a large dataset like ABIDE for multi-group analysis. PALM can shuffle image observations with the use of complex, tree-like covariance structure for permutations. 


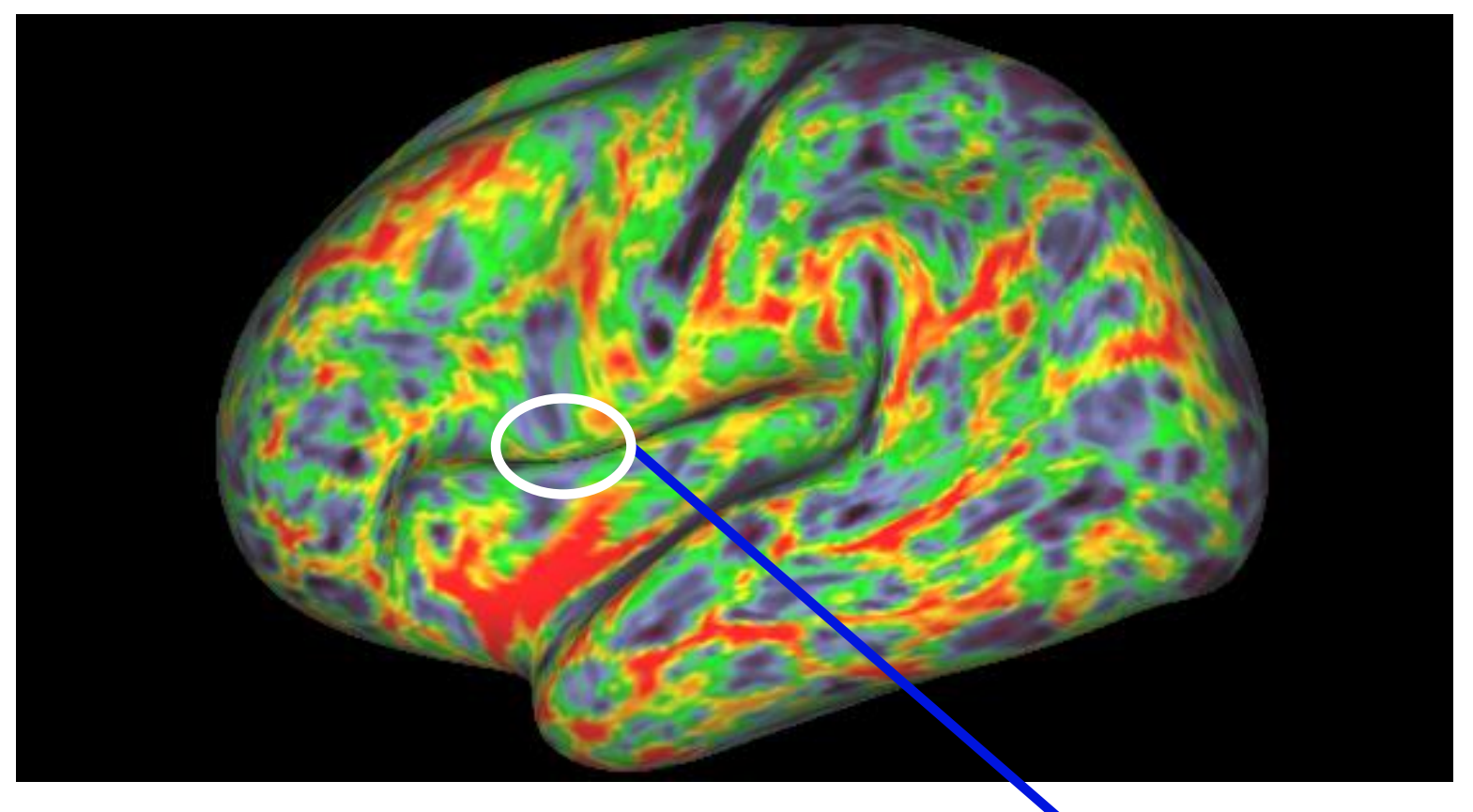

Figure 11: The surface can be separated using different vertices called "grayordinates" (right image). Here, each grayordinate contains information about how thick the cortex is at that location (image above)

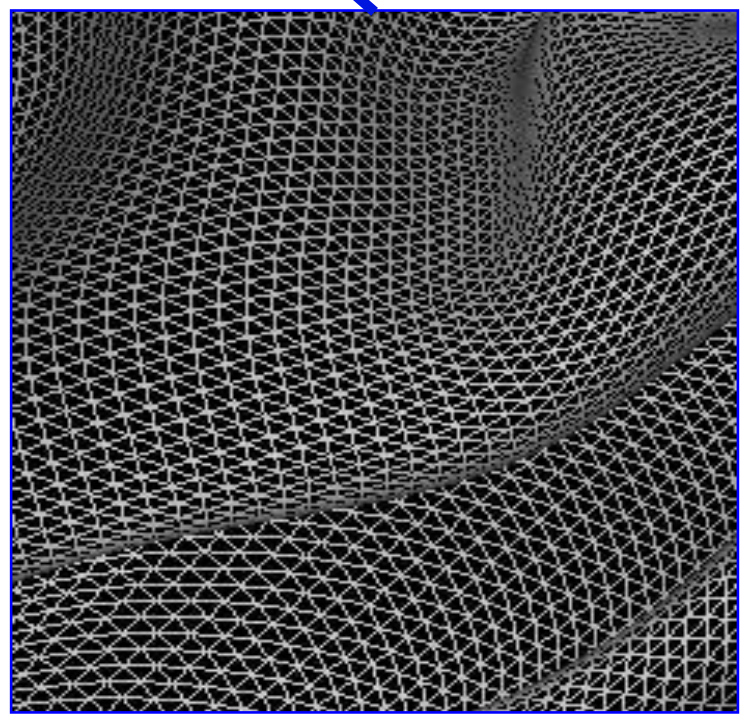

MATLAB, a high-performance computing platform, was used to execute PALM. The models are generated using the spatial information provided by grayordinates in the subject's CIFTI files. Cluster detection of cortical thickness differences between ASD and TD groups is done by converting p-values to log-transformed p-values in Eq. 1 to ignore any insignificant results. 
[Eq. 1] $-\log (p-$ value $)=\log$ transformed $p-$ value

Using a p-value of 0.027 will result in a log-transformed $p$-value of 1.56: $-\log (0.027)=$ 1.56. A threshold can be set to identify and calculate cluster size. Once all the parameters are set, permutation tests can be performed to determine any surface area or volumetric differences within different brain regions.

Reprocessed subjects were separated into ASD and TD groups. Subject files along with directories were created using MATLAB. Once files are generated, the PALM software can be activated with MATLAB from within the software itself, from a shell, or scripts. Once activated it will perform permutation tests on both subject groups. The generated cortical thickness scans can be viewed using workbench (https://dev.mysql.com/doc/workbench/en/).

A parcellation scheme devised by Gordon et al. was used to distinguish certain functional brain systems of the brain (Gordon et al., 2016). Statistic maps will be present within the cortical thickness scans to denote any differences in cortical thickness between the ASD and TD subgroups.

\section{Results}

The following figures are cortical thickness images generated with PALM using the reprocessed ABIDE dataset. The PALM results are presented in Figures 12-14. These are all images of a representative brain, showing statistical differences between groups but in different orientations. The brain outputs are also color-coded to include functional network 
markings (based on the Gordon Parcellation) to highlight the functional brain systems where these differences lie. Refer to Table 1 for all functional network labels.

\section{Table 1: Gordon Functional Network Parcellation}

\begin{tabular}{|c|c|}
\hline Color & Functional Network \\
\hline Red & Default \\
\hline Blue & Visual \\
\hline Magenta & Auditory \\
\hline Cyan & Somatomotor Hand \\
\hline Orange & Somatomotor Mouth \\
\hline Green & Dorsal Attention Network \\
\hline Purple & Cingulo-Opercular Network \\
\hline Yellow & Frontoparietal network \\
\hline Turquoise & Ventral Attention Network \\
\hline
\end{tabular}

The heat map on the bottom left of Figures 12-14 shows the log-transformed inverse $p$ value to signify any differences of cortical thickness between ASD and TD subjects provided by the ABIDE dataset. All the ASD data underwent PALM analysis, and the same was done for the TD subjects. PALM was able to generate an average of ASD and TD brains so we can measure the cortical thickness differences between both subgroups. 


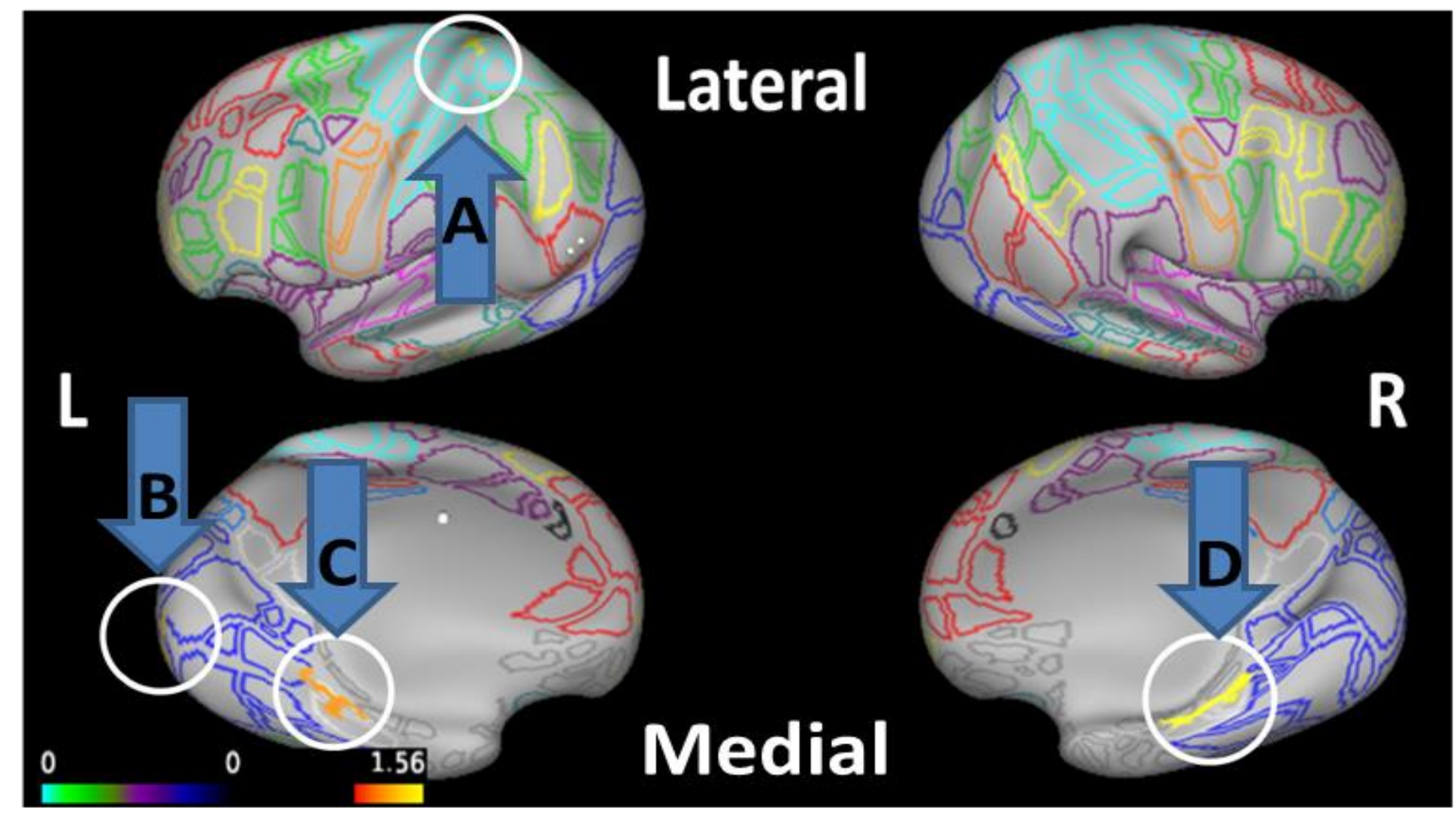

Figure 12: Lateral and Medial View of PALM scan. The top two brain images display the lateral portion of the brain, and the bottom two brain scans showcase the medial portion 


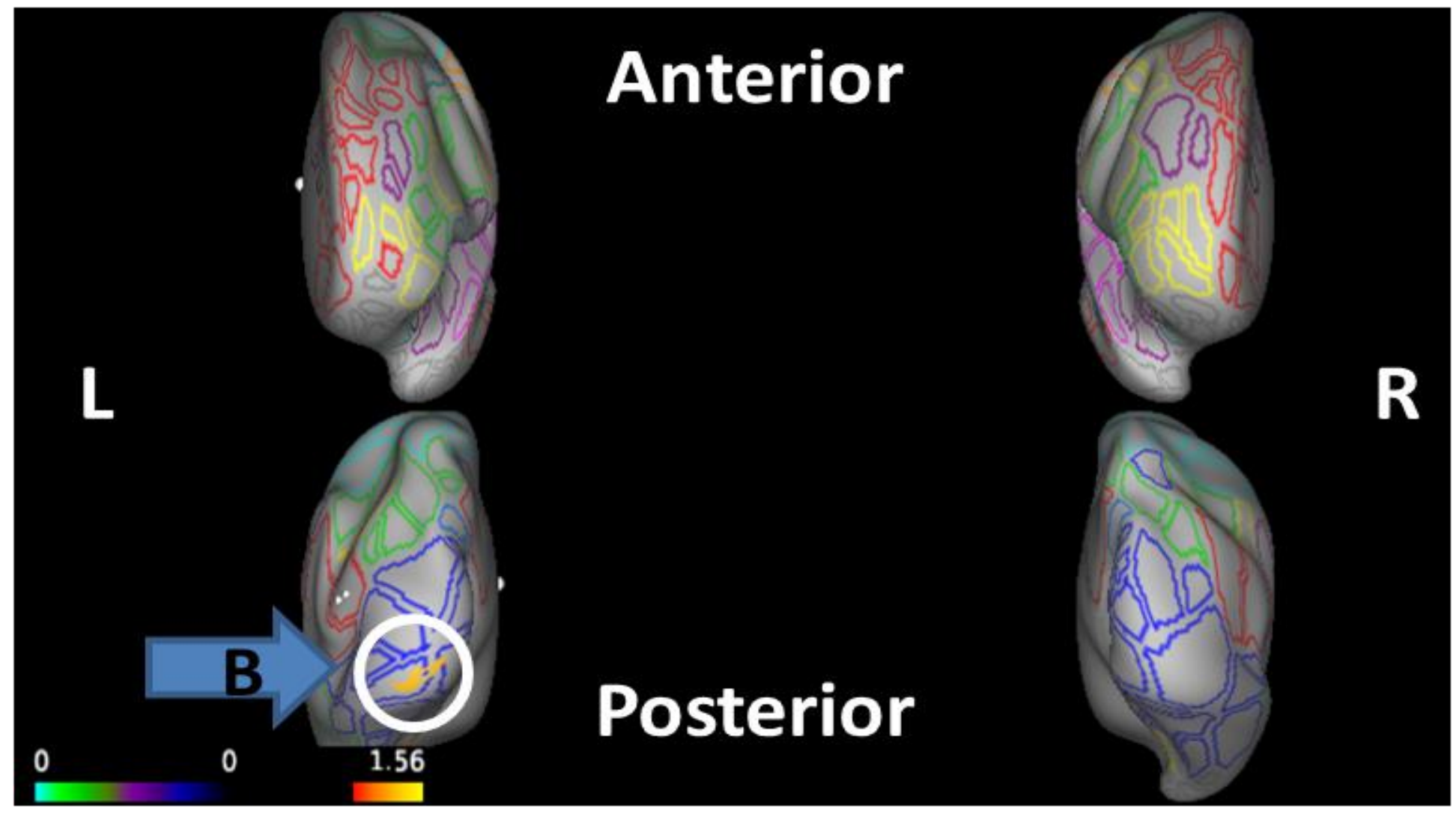

Figure 13: Anterior and Posterior View of PALM Scan. The top two images show the anterior portion of the brain, and the bottom two images represent the posterior portion of the brain 


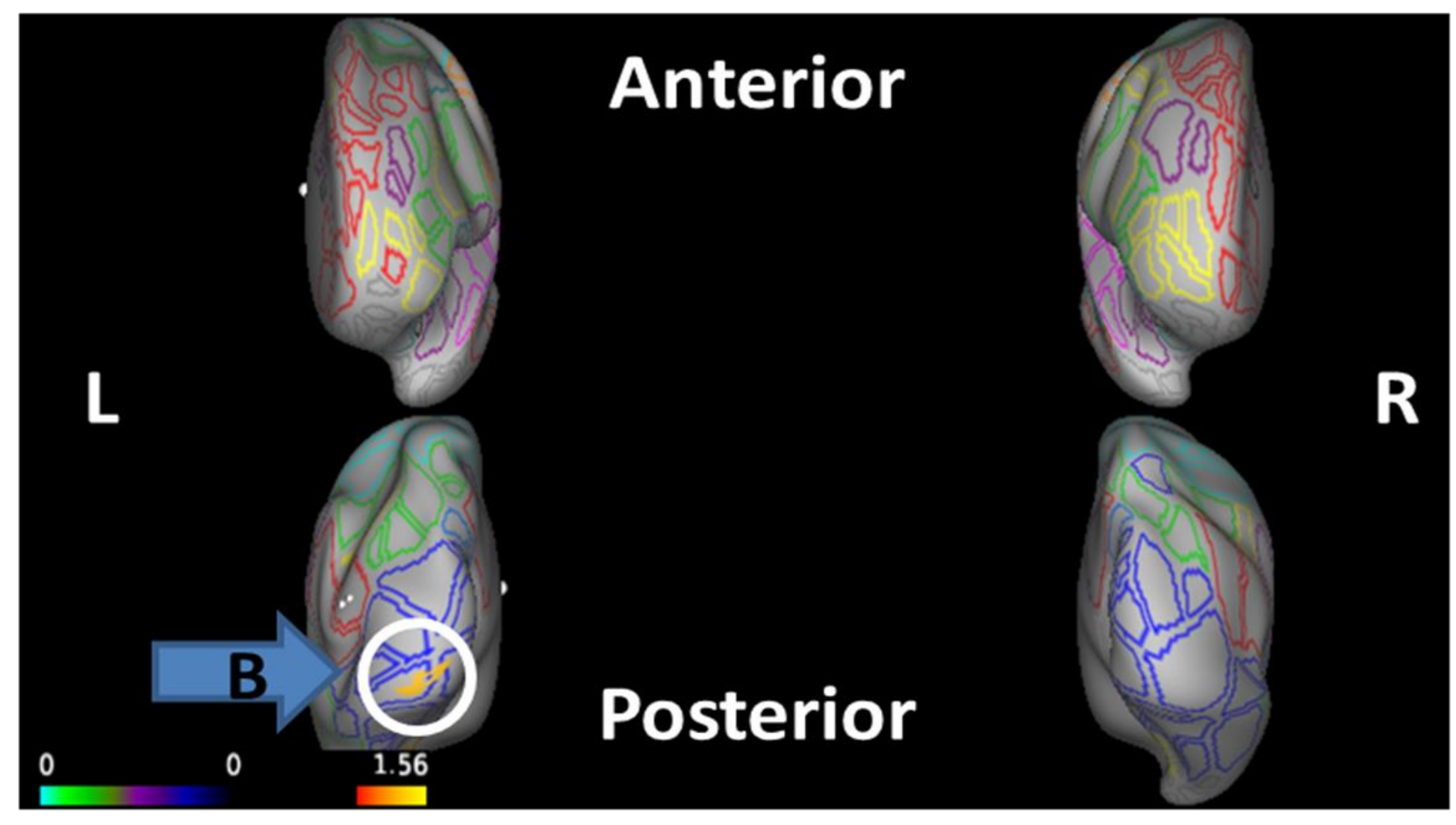

Figure 14: Anterior and Posterior View of PALM Scan. The top two images show the anterior portion of the brain, and the bottom two images represent the posterior portion of the brain 
The sagittal, coronal, and transversal views are shown in Figures 12, 13, and 14 respectively. Each figure contains ROls that are orange or yellow which show statistical differences between ASD and TD brains. All statistical differences are scaled as an inverse $\log p$ value (see arrows). Table 2 contains information about the anatomical, and functional region of each ROI as well as their p-values and cluster sizes.

\section{Table 2: ROI Results}

\begin{tabular}{c|c|c|c|c|c}
\hline Label & Anatomical Label & Functional Label & Log P & P-value & Cluster size $\left(\mathrm{mm}^{2}\right)$ \\
\hline A & Left Superior Parietal & Somatomotor Hand & 1.248 & 0.0565 & 202.75 \\
B & Left Occipital Lobe & Visual Cortex & 1.065 & 0.0861 & 175.13 \\
C & Left Temporal Lobe & Visual Cortex & 1.235 & 0.0582 & 202.76 \\
D & Right Temporal Lobe & Undefined; Visual Cortex & 1.562 & 0.0274 & 265.5 \\
\hline
\end{tabular}

ROI " $A$ " is located in the left superior parietal involved in somatomotor hand movements $\left(p=0.0565\right.$; cluster size $\left.=202.75 \mathrm{~mm}^{2}\right)$. The left visual cortex contains two ROIs. In the left occipital lobe you see region " $B$ " $\left(p=0.0861\right.$; cluster size $\left.=175.13 \mathrm{~mm}^{2}\right)$, and in the left temporal lobe you see region " $C$ " $\left(p=0.0582\right.$; cluster size $\left.=202.76 \mathrm{~mm}^{2}\right)$. The final ROI, region “ $D$ ” ( $p=0.0274$; cluster size $\left.=265.5 \mathrm{~mm}^{2}\right)$, is the largest and most statistically significant ROI found in the right temporal lobe. Part of region " $D$ " is within the visual cortex and an undefined functional network of the brain. All these regions show an increase in overall cortical thickness. 


\section{Discussion}

The diagnosis and symptoms of ASD have been well researched and documented, but the biomarkers to verify an ASD diagnosis has not been well established. The purpose of this research was to show the differences in brain structure between ASD and TD subjects using measurements of cortical thickness. Cluster regions show cortical thickness differences in the left superior parietal $\left(p=0.0565\right.$; cluster size $\left.=202.75 \mathrm{~mm}^{2}\right)$, left occipital lobe $(p=0.0861$; cluster size $\left.=175.13 \mathrm{~mm}^{2}\right)$, left temporal lobe $\left(p=0.0582\right.$; cluster size $\left.=202.76 \mathrm{~mm}^{2}\right)$, and the right temporal lobe $\left(p=0.0274\right.$; cluster size $\left.=265.5 \mathrm{~mm}^{2}\right)$. All these results showcase an increase in cortical thickness in ASD subjects.

These results are consistent with other research findings that show the unique characteristics of ASD involved in motor and sensory areas. Visual processing is different in ASD than TD individuals and the difference in cortical thickness in the visual and motor cortex can explain the enhanced detail processing in ASD (Vandenbroucke, Steven Scholte, Engeland, Lamme, \& Kemner, 2009). The temporal and occipital regions of the brain are involved in face processing and mental state attributions, and the results showcase cortical thickness increases in both temporal lobes ( $\mathrm{ROI}$ " $\mathrm{C}$ " and " $\mathrm{D}$ ") along with the left occipital lobe (ROI " $\mathrm{B}$ "). The motor cortex abnormalities are supported by ROI " $\mathrm{A}$ " in the left superior parietal which affects the somatomotor hand movements. The combination of visual and motor cortex thickness differences could explain the poor integration of visual input and motor output of ASD individuals (Nebel et al., 2016).

The reason(s) for an increase in cortical thickness in the identified ROls are unknown. The increase could be due to an increase in the number of glial cells in these areas, or an 
increase in the amount of gray and white matter. The future direction for this project is to incorporate the reprocessed ABIDE dataset with different software to further validate these results, and to test the correlation between symptoms of ASD with cortical thickness. The PALM software can show the cortical thickness difference, but this software is still experimental and requires further refinement. PALM and other software packages still rely on having clean usable data to generate accurate and reproducible results. To address these issues using MRI machines with stronger magnets such as a $3 \mathrm{~T}$ or $7 \mathrm{~T}$ machines, while performing a standardized protocol to ensure proper data acquisition, can improve the number of usable scans available. Motion is a strong variable that can cause neuroimaging artifacts that can affect the subject's scan quality. Reducing the amount of motion by the subject within the scanner will help increase the likely-hood of proper data acquisition. Further improvements to the DCAN modified HCP pipeline to account for denoising, bias field correction, and motion correction can be done to remove any possible artifacts within the subject's scans. The next goal for this reprocessed ABIDE dataset is to release it in a publicly available open-source repository to ensure reliable data is made available to neuroscientists everywhere, so that they may use it for ASD research.

\section{Acknowledgments}

I would like to thank the entire DCAN Labs members at Oregon Health and Science University for their guidance and support. Dr. Eric Feczko for his assistance with the statistical analysis, the DCAN computing team for their pipeline processing assistance, and Dr. Damien Fair for being my thesis advisor. I also thank the BUILD EXITO and the Robert E. McNair scholar programs at Portland State University. 
Outside of my academic circles, I express my gratitude to all my family and friends. My

best friend Jose helped me finish my degree in Portland by moving out with me and being my roommate. He was there for my celebrations and my downfalls these past few years. My siblings Randy, Richard, and Shanty blessed me with the opportunity of being the big brother of the family. They taught me responsibility, discipline, faith, happiness, and loyalty. They are a huge reason why I am the person I am today. Lastly, I give all the love in the world to my mother Mirna. She believed in me long before anyone else. Being the first person in the family to graduate from college is one of the many ways I can repay her for all she has given me.

\section{References}

American Psychiatric Association. (2013). Diagnostic and statistical manual of mental disorders : DSM-5. DSM-5 (5th ed.). Arlington, VA: Arlington, VA : American Psychiatric Association.

Avants, B. B., Tustison, N., \& Song, G. (2009). Advanced Normalization Tools (ANTS). Insight Journal, 1-35. https://doi.org/http://hdl.handle.net/10380/3113

Baio, J., Wiggins, L., Christensen, D. L., Maenner, M. J., Daniels, J., Warren, Z., ... Dowling, N. F. (2018). Prevalence of Autism Spectrum Disorder Among Children Aged 8 Years - Autism and Developmental Disabilities Monitoring Network, 11 Sites, United States, 2014. Morbidity and Mortality Weekly Report. Surveillance Summaries (Washington, D.C. : 2002), 67(6).

Ciric, R., Wolf, D. H., Power, J. D., Roalf, D. R., Baum, G. L., Ruparel, K., ... Satterthwaite, T. D. (2017). Benchmarking of participant-level confound regression strategies for the control of motion artifact in studies of functional connectivity. Neurolmage, 154(March), 174-187. https://doi.org/10.1016/j.neuroimage.2017.03.020

Craig, F., Lamanna, A. L., Margari, F., Matera, E., Simone, M., \& Margari, L. (2015). Overlap Between Autism Spectrum Disorders and Attention Deficit Hyperactivity Disorder: Searching for Distinctive/Common Clinical Features. Autism Research, 8(3), 328-337. https://doi.org/10.1002/aur.1449

Despotović, I., Goossens, B., \& Philips, W. (2015). MRI segmentation of the human brain: Challenges, methods, and applications. Computational and Mathematical Methods in Medicine, 2015. https://doi.org/10.1155/2015/450341

Di Martino, A., Yan, C. G., Li, Q., Denio, E., Castellanos, F. X., Alaerts, K., ... Milham, M. P. (2014). The autism brain imaging data exchange: Towards a large-scale evaluation of the intrinsic brain architecture in autism. Molecular Psychiatry, 19(6), 659-667.

https://doi.org/10.1038/mp.2013.78 
Ecker, C. (2012). Brain Anatomy and Its Relationship to Behavior in Adults With Autism Spectrum Disorder. Archives of General Psychiatry, 69(2), 195. https://doi.org/10.1001/archgenpsychiatry.2011.1251

Eklund, A., Nichols, T. E., \& Knutsson, H. (2016). Cluster failure: Why fMRI inferences for spatial extent have inflated false-positive rates. Proceedings of the National Academy of Sciences of the United States of America, 113(28).

Evans, A. C., Janke, A. L., Collins, D. L., \& Baillet, S. (2012). Brain templates and atlases. Neurolmage, 62(2), 911-922. https://doi.org/10.1016/j.neuroimage.2012.01.024

Frazier, T. W., Youngstrom, E. A., Speer, L., Embacher, R., Law, P., Constantino, J., ... Eng, C. (2012). Validation of proposed DSM-5 criteria for autism spectrum disorder. Journal of the American Academy of Child and Adolescent Psychiatry, 51(1), 28-40.e3. https://doi.org/10.1016/j.jaac.2011.09.021

Giedd, J. N., Lalonde, F. M., Celano, M. J., White, S. L., Wallace, G. L., Lee, N. R., \& Lenroot, R. K. (2009). Anatomical brain magnetic resonance imaging of typically developing children and adolescents. Journal of the American Academy of Child and Adolescent Psychiatry, 48(5), 465-470. https://doi.org/10.1097/CHI.0b013e31819f2715

Glasser, M. F., Sotiropoulos, S. N., Wilson, J. A., Coalson, T. S., Fischl, B., Andersson, J. L., ... Jenkinson, M. (2013). The minimal preprocessing pipelines for the Human Connectome Project. Neurolmage, 80, 105-124. https://doi.org/10.1016/j.neuroimage.2013.04.127

Gordon, E. M., Laumann, T. O., Adeyemo, B., Huckins, J. F., Kelley, W. M., \& Petersen, S. E. (2016). Generation and Evaluation of a Cortical Area Parcellation from Resting-State Correlations. Cerebral Cortex, 26(1), 288-303. https://doi.org/10.1093/cercor/bhu239

Haar, S., Berman, S., Behrmann, M., \& Dinstein, I. (2016). Anatomical Abnormalities in Autism? Cerebral Cortex, 26(4), 1440-1452. https://doi.org/10.1093/cercor/bhu242

Johnson, C. P., Myers, S. M., Lipkin, P. H., Cartwright, J. D., Desch, L. W., Duby, J. C., ... YearginAllsopp, M. (2007). Identification and evaluation of children with autism spectrum disorders. Pediatrics, 120(5), 1183-1215. https://doi.org/10.1542/peds.2007-2361

Koolschijn, P. C. M. P., \& Geurts, H. M. (2016). Gray Matter Characteristics in Mid and Old Aged Adults with ASD. Journal of Autism and Developmental Disorders, 46(8), 2666-2678. https://doi.org/10.1007/s10803-016-2810-9

Lord, C., Rutter, M., DiLavore, P., Risi, S., \& Gotham, K. (2012). Autism diagnostic observation schedule, (ADOS-2). Los Angeles, California.

Lord, C., Rutter, M., \& Couteur, A. L. (1994). ADIR.pdf. Journal of Autism and Developmental Disorders, 24(5), 659-685.

Matson, J. L., Matson, M. L., \& Rivet, T. T. (2007). Social-Skills Treatments for Children With Autism Spectrum Disorders: An Overview. Behavior Modification, 31(5), 682-707.

Nebel, M. B., Eloyan, A., Nettles, C. A., Sweeney, K. L., Ament, K., Ward, R. E., ... Mostofsky, S. H. (2016). Intrinsic visual-motor synchrony correlates with social deficits in autism. Biological Psychiatry, 79(8), 633-641. https://doi.org/10.1016/j.biopsych.2015.08.029

Nordahl, C. W., Braunschweig, D., losif, A. M., Lee, A., Rogers, S., Ashwood, P., ... Van de Water, J. (2013). Maternal autoantibodies are associated with abnormal brain enlargement in a subgroup of children with autism spectrum disorder. Brain, Behavior, and Immunity, 30, 61-65. https://doi.org/10.1016/j.bbi.2013.01.084

Ousley, O., \& Cermak, T. (2014). Autism Spectrum Disorder: Defining Dimensions and 
Subgroups. Current Developmental Disorders Reports, 1(1), 20-28.

https://doi.org/10.1007/s40474-013-0003-1

Pagnozzi, A. M., Conti, E., Calderoni, S., Fripp, J., \& Rose, S. E. (2018). A systematic review of structural MRI biomarkers in autism spectrum disorder: A machine learning perspective. International Journal of Developmental Neuroscience, 71(August), 68-82. https://doi.org/10.1016/j.ijdevneu.2018.08.010

Raichle, M. E., \& Mintun, M. A. (2006). Brain Work and Brain Imaging. Annual Review of Neuroscience, 29(1), 449-476. https://doi.org/10.1146/annurev.neuro.29.051605.112819

Rosen, T. E., Mazefsky, C. A., Vasa, R. A., \& Lerner, M. D. (2018). Co-occurring psychiatric conditions in autism spectrum disorder. International Review of Psychiatry, 30(1), 40-61. https://doi.org/10.1080/09540261.2018.1450229

Savalia, N. K., Agres, P. F., Chan, M. Y., Feczko, E. J., Kennedy, K. M., \& Wig, G. S. (2017). Motionrelated artifacts in structural brain images revealed with independent estimates of inscanner head motion. Human Brain Mapping, 38(1), 472-492. https://doi.org/10.1002/hbm.23397

Siegel, J. S., Power, J. D., Dubis, J. W., Vogel, A. C., Church, J. A., Schlaggar, B. L., \& Petersen, S. E. (2014). Statistical improvements in functional magnetic resonance imaging analyses produced by censoring high-motion data points. Human Brain Mapping, 35(5), 1981-1996. https://doi.org/10.1002/hbm.22307

Strang, J. F., Kenworthy, L., Daniolos, P., Case, L., Wills, M. C., Martin, A., \& Wallace, G. L. (2012). Depression and anxiety symptoms in children and adolescents with autism spectrum disorders without intellectual disability. Research in Autism Spectrum Disorders, 6(1), 406-412. https://doi.org/10.1016/j.rasd.2011.06.015

Strimbu, K., \& Tavel, J. A. (2010). What are biomarkers? Current Opinion in HIV and AIDS, 5(6), 463-466. https://doi.org/10.1097/COH.0b013e32833ed177

Tager-Flusberg, H., \& Caronna, E. (2007). Language Disorders: Autism and Other Pervasive Developmental Disorders. Pediatric Clinics of North America, 54(3), 469-481. https://doi.org/10.1016/j.pcl.2007.02.011

Vandenbroucke, M. W. G., Steven Scholte, H., Engeland, H. van, Lamme, V. A. F., \& Kemner, C. (2009). A new approach to the study of detail perception in Autism Spectrum Disorder (ASD): Investigating visual feedforward, horizontal and feedback processing. Vision Research, 49(9), 1006-1016. https://doi.org/10.1016/j.visres.2007.12.017

Winkler, A. M., Ridgway, G. R., Webster, M. A., Smith, S. M., \& Nichols, T. E. (2014). Permutation inference for the general linear model. Neurolmage, 92, 381-397. https://doi.org/10.1016/j.neuroimage.2014.01.060 